\title{
On the performance of distributed space-time coded cooperative relay networks based on inter-relay communications
}

\author{
Le-Quang-Vinh Tran*, Olivier Berder and Olivier Sentieys
}

\begin{abstract}
A new protocol, called fully distributed space-time coded (FDSTC) protocol having information exchange between relays, is proposed and compared with the conventional distributed space-time coded (DSTC) protocol using non-regenerative relays (NR-relays) and regenerative relays (R-relays). Closed-form error probabilities are derived to verify the simulations. In terms of error performance, the FDSTC protocol gets significant average signal-to-noise ratio (SNR) gains (3.7 dB for NR-relays and $18.1 \mathrm{~dB}$ for R-relays). In addition, the impact of the relative distance of relays on the required SNR is reduced up to 70\%. The system diversity order using the FDSTC protocol is higher than that using the DSTC protocol (especially, the FDSTC protocol obtains full diversity with NR-relays). As a result, at the same spectral efficiency, FDSTC has better performance in terms of outage probability in high SNR regions. In terms of energy efficiency, the FDSTC protocol is shown to outperform DSTC for long-range transmissions.
\end{abstract}

\section{Introduction}

In the last years, cooperative relay has been identified as a core technique to overcome challenges in wireless environments. Based on the transmission protocol performed at relays, we can classify them into two main categories: repetition-based and distributed space-time code-based (DSTC-based) cooperative relay techniques [1]. In repetition-based cooperative relay techniques, each relay, after receiving signal from the source, will consecutively forward the signal to destination. Each transmitted signal (including the original one transmitted from source to destination) is passed through multiple independent paths, and thus, the probability that the signal fails to reach the destination is significantly reduced. In contrast to repetition-based cooperative relay techniques, in DSTC-based cooperative relay techniques, the relays will take advantage of DSTC techniques to simultaneously forward the signal to the destination, and hence, the spectral efficiency of the system is increased. The initial work on using DSTC protocols over relay channel is considered in [1-4]. We denote them as the conventional DSTC protocols, in which the whole communication consists of only

\footnotetext{
*Correspondence: vinh.tran@irisa.fr
}

INRIA/IRISA, Universite de Rennes 1, 6 rue de Kerampont, Lannion 22300, France two phases. Firstly, the source transmits signals to relays and destination. Secondly, the non-regenerative or regenerative relays (NR-relays or R-relays, respectively) use the DSTC protocol to forward signals to the destination.

\subsection{Related work}

Recently, data exchanges among the relays, or interrelay communications, have been considered in [5-9]. In [5], an inter-relay cooperation scheme allowing message exchanges among the relays was presented in order to mitigate the impact of error propagation of R-relays. A hybrid TDMA-FDMA-based protocol with inter-relay communication for Nakagami-m fading channel was investigated in [6]. The lower bound on outage probability of the interrelay communication-based protocol was mathematically derived and validated by simulations [7]. Besides, the novel idea of inter-relay traffic cooperative network-coded hybrid ARQ was proposed as a smart way to improve R-relays in [8].

Error probability of DSTC protocols was derived for NR-relays in [4,10-13]. An approximate formula of average symbol error probability (ASEP) for a DSTC system based on multi-user cooperation was found in [4]. On the other hand, performance of the DSTC protocol in regenerative relay networks was derived in [3,14-17]. In [3], a regenerative DSTC protocol is applied for source and

\section{Springer}

(C) 2013 Tran et al: licensee Springer. This is an Open Access article distributed under the terms of the Creative Commons Attribution License (http://creativecommons.org/licenses/by/2.0), which permits unrestricted use, distribution, and reproduction in any medium, provided the original work is properly cited. 
relay with optimal allocation of transmit power in order to minimize the average bit error rate (BER) at final destination. In [17], Anghel et al. proposed two error-aware distributed space-time (EADST) protocols to overcome worse BER performance of R-relays induced by decoding errors at the relays. However, these two protocols require feedback from destination. Recently, an extension of the DSTC protocols for more than two hops was considered in [18].

Besides BER performance consideration, in [2], the DSTC protocol was shown to be effectively used for higher spectral efficiency and to achieve full spatial diversity in some specific cases. In [1], the performance of cooperative protocols was considered in terms of outage probability. The authors showed that, except for fixed decode-andforward (DF) protocol, all of their cooperative protocols are efficient in the sense of achievement of full diversity. In [13], the DSTC system was shown to have the same diversity as a multiple-antenna system when the coherence interval is greater than the number of relays. With NR-relays, the diversity of the DSTC system was shown to depend on the scaling factor of the relays [12]. In [19], a diversity-multiplexing trade-off (DMT) analysis was considered in multi-hop multiple-input and multiple-output (MIMO) relay networks. A DMT analysis was also considered recently for an AF two path half-duplex relaying scheme in [20]. Wicaksana showed that DMT is achievable for finite codeword lengths with a careful choice of coding strategy.

Cooperative techniques have recently been potential candidates to reduce energy consumption in wireless networks. In [21], using cooperative MIMO systems, a tremendous energy saving was shown for long transmission distances in comparison with single-input and singleoutput (SISO) systems. Besides, the energy efficiency of different cooperative relaying techniques was investigated. In LTE-advanced networks, using relay nodes, it was shown that energy saving up to $15.6 \%$ is possible in the two hop schemes, and up to $8.5 \%$ are possible with the multicast cooperative scheme [22]. In [23], a transparent relay with cooperative strategy was shown to save about $60 \%$ power consumption as compared to the transparent relay in IEEE 802.16j under given simulation configurations.

Inter-relay communications have been considered in the repetition-based cooperative relay protocol and are shown to be able to increase its performances [5-8]. The present paper associate inter-relay communications with the DSTC-based cooperative relay protocol to further increase its performance. The fully distributed space-time coded (FDSTC) protocol in an inter-relay communication phase is added for cooperative relay systems. FDSTC was originally mentioned in [9] with NR-relays jointly with a lower bound on its ASEP. In this work, the FDSTC protocol is thoroughly considered in terms of error probability, outage probability, diversity order, and energy efficiency. Firstly, a closed-form ASEP of non-regenerative relays based on FDSTC is considered. Moreover, for regenerative relays, a closed-form expression of error probability, conditioned to the channel, is also derived. With the help of data exchange between relays in the FDSTC protocol, the SNR at relays is increased. This leads to a higher SNR gain obtained in comparison with conventional DSTC protocols, where there is no data exchange between the relays. Secondly, the present paper also considers the outage probability of the protocols to have a fair comparison in terms of data rate. At the same spectral efficiency, the FDSTC protocol is shown to have better performance in high SNR regions due to its higher system diversity order. Using automatic gain control (AGC) at the relays, the same result as in [12] is found, i.e., a conventional DSTC system cannot get full diversity. By contrast, the FDSTC system is shown to achieve full diversity in case of NR-relays. Finally, since in the FDSTC protocol the relays also need power to exchange data, it is very important to consider the total power consumption of the protocols to have a fair comparison. Using a typical energy model as in [21], the FDSTC protocol is shown to be able to get energy efficiency for long-range transmissions.

The rest of this paper is organized as follows. The system model is thoroughly described in Section 2. In Section 3, the error performance of FDSTC protocol with NR-relays and R-relays is analyzed through mathematical expressions. Outage probability and DMT are considered in Section 4 to evaluate the protocol performance in terms of spectral efficiency. Section 5 presents and compares energy efficiency of the protocols. Numerical results are derived in Section 6. Finally, conclusion is given in Section 7.

Throughout the paper, bold upper (lower) case denotes a matrix (column vector). Superscript $T$ stands for transpose. $\operatorname{Re}\{x\}$ stands for the real part of a complex number $x$.

\section{System model}

In this paper, a cooperative relay system including one source, one destination, and two relays which have all only one antenna (Figure 1) is considered to exploit the FDSTC protocol. In the system, the relays only play a role to enhance the quality of the source-destination link. They do not have their own data to transmit. In order to avoid self-interference, the relays are used in half-duplex mode, which means they can not transmit and receive data simultaneously. The ideal channel state information (CSI) is known at the receiver but not at the transmitters. A binary phase shift keying (BPSK) modulation is considered for all the analysis and simulations. We assume that all communications are performed over a flat Rayleigh fading channel. Channel coefficients remain the same for 


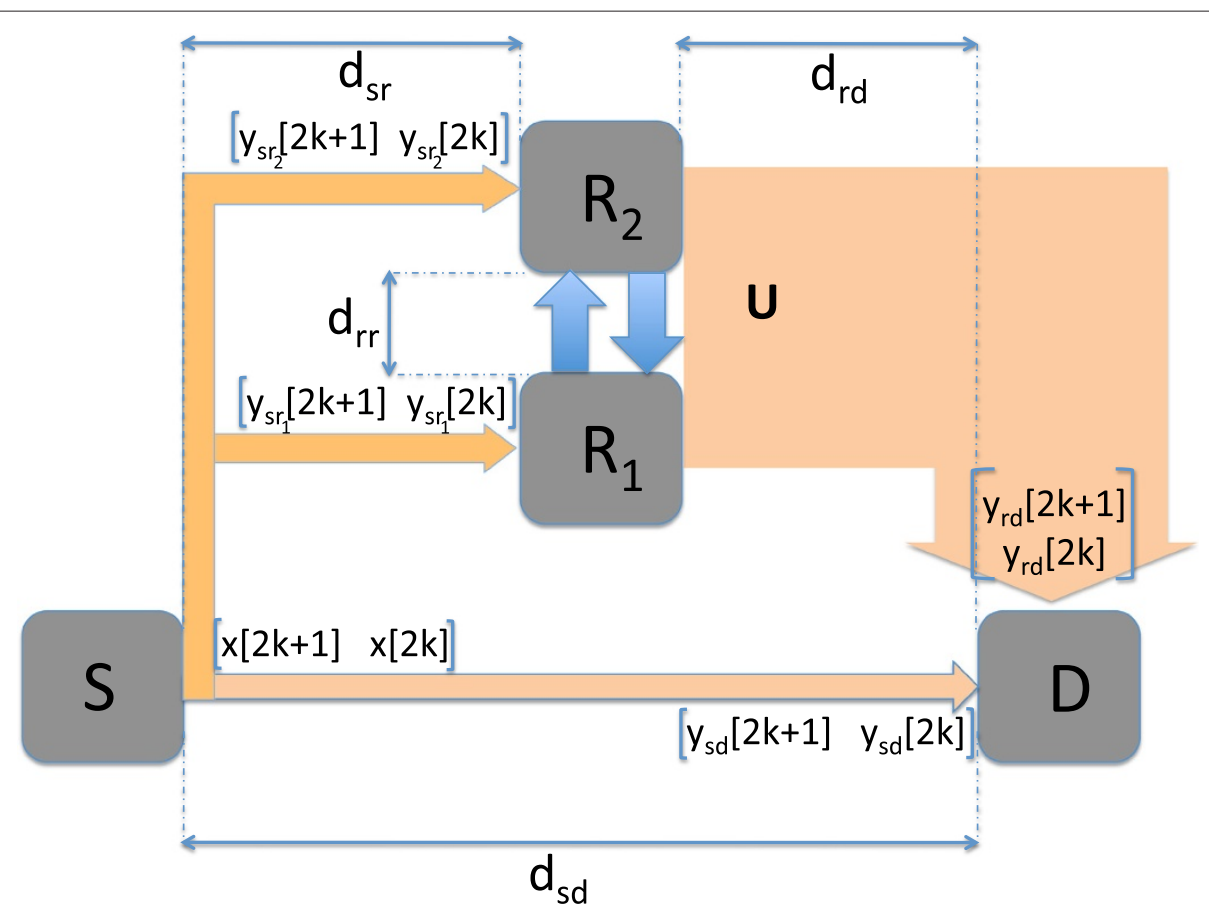

Figure 1 FDSTC protocol for a system including one source (S), two relays (R1, R2), and one destination (D). Source-relay distance, source-destination distance, relay-destination distance, and relay-relay distance are respectively denoted as $d_{\mathrm{sr}}, d_{\mathrm{sd}}, d_{\mathrm{rd}}$, and $d_{\mathrm{rr}}$.

two consecutive time intervals. Statistically, we model the channel coefficients $h_{i j}[n]$ with $i \in\left\{s, r_{1}, r_{2}\right\}, j \in\left\{r_{1}, r_{2}, d\right\}$, and $i \neq j$ as zero mean, independent, complex Gaussian random variables with variances $\Omega_{i j}$. Similarly, we model additive white Gaussian noise (AWGN) $z_{i j}[n]$ as zero mean mutually independent complex Gaussian random variables with variances $N_{i j}$. For the clarity of the following expressions, the symbols $h_{i j}[n], G_{i j}[n], \gamma_{i j}[n]$ will be rewritten in brief form of $h_{i j}, G_{i j}, \gamma_{i j}$.

The first idea of the FDSTC protocol is presented in [9] with NR-relays by deriving the bounds on error performances. However, the present paper will give thorough considerations of the FDSTC protocol with both NRrelays and R-relays by deriving closed-form error performance and considering outage probability, diversity order, and energy efficiency as well.

The FDSTC protocol can be mathematically described as below. In the first phase, the source transmits signals $\boldsymbol{x}=[x[2 k] x[2 k+1]]^{T}\left(E[x[n]]=0\right.$, and $\left.E\left[x^{2}[n]\right]=1\right)$ with the transmit power $\epsilon_{s}=\epsilon_{\mathrm{sd}}=\epsilon_{\mathrm{sr}_{1}}=\epsilon_{\mathrm{sr}_{2}}$ to the relays and the destination at the same time. The received signals at the relays and the destination (Figure 1) can be represented as $\boldsymbol{y}_{\mathrm{sr}_{1}}, \boldsymbol{y}_{\mathrm{sr}_{2}}$, and $\boldsymbol{y}_{\mathrm{sd}}$, respectively

$$
\boldsymbol{y}_{s j}=\sqrt{\epsilon_{s j}} h_{s j} \boldsymbol{x}+\boldsymbol{z}_{s j}, \quad j \in\left\{r_{1}, r_{2}, d\right\},
$$

where $\boldsymbol{y}_{s j}=\left[y_{s j}[2 k] y_{s j}[2 k+1]\right]^{T}, \boldsymbol{z}_{i j}=\left[z_{i j}[2 k] z_{i j}\right.$ $[2 k+1]]^{T}$ and $\epsilon_{s j}$ is the transmit power of the source to the terminal $j$.
In the second phase, the two relays exchange their data with each other. The received signals at relay $R_{1}$ from relay $R_{2}$ and vice versa respectively symbolized as $\boldsymbol{y}_{r_{2} r_{1}}$, and $\boldsymbol{y}_{r_{1} r_{2}}$, are given by

$$
\boldsymbol{y}_{i j}=h_{i j} G_{s i} \boldsymbol{y}_{s i}+\boldsymbol{z}_{i j}, \quad i, j \in\left\{r_{1}, r_{2}\right\}, i \neq j,
$$

where $G_{s i}$ is the AGC which prevents saturating the relay $i$ and can be chosen as $G_{s i}=\sqrt{\frac{\epsilon_{i j}}{\epsilon_{s}\left|h_{s i}\right|^{2}+N_{s i}}}, \quad i, j \in$ $\left\{r_{1}, r_{2}\right\}, i \neq j$, with $\epsilon_{r r}=\epsilon_{r_{1} r_{2}}=\epsilon_{r_{2} r_{1}}$ the transmit power between the relays. Traditionally, in dense wireless sensor networks (WSNs), the distance between two relays is very small in comparison with the transmission distance. Therefore, to save energy, the transmit power between the relays can be chosen as one tenth of the transmit power from the relays to the destination (i.e., $\epsilon_{\mathrm{rr}}=$ $\left.1 / 10 \epsilon_{s}\right)$. As a result, the destination cannot overhear the inter-communication between the relays.

Then, each relay uses the maximum ratio combining (MRC) technique to combine the signals received from the source and from the other relay

$$
\boldsymbol{u}_{j}=\frac{h_{s j}^{*} \sqrt{\epsilon_{s j}}}{N_{s j}} \boldsymbol{y}_{s j}+\frac{\sqrt{\epsilon_{s j}} h_{i j}^{*} G_{s i}^{*} h_{s i}^{*}}{\left|h_{i j}\right|^{2}\left|G_{s i}\right|^{2} N_{s i}+N_{i j}} \boldsymbol{y}_{i j}
$$

where $i, j \in\left\{r_{1}, r_{2}\right\}, i \neq j, \boldsymbol{y}_{i j}=\left[y_{i j}[2 k] \quad y_{i j}[2 k+1]\right]^{T}$, and $\boldsymbol{u}_{j}=\left[u_{j}[2 k] u_{j}[2 k+1]\right]^{T}$.

In the last phase, the two relays use distributed spacetime codes to simultaneously transmit the Alamouti reencoded signals $U$ to the destination (see Section 3.1 for 
more details). In this work, Alamouti space-time code is used, thanks to its simplicity and full rate capacity. A consideration of other space-time codes [24-26] is out of the scope of this paper. The components of the matrix $U$ depend on the protocol that the relays use to forward the signals to the destination: non-regenerative or regenerative. The signals received at the destination from the

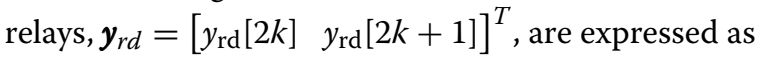

$$
\boldsymbol{y}_{\mathrm{rd}}=\boldsymbol{U} \boldsymbol{h}_{\mathrm{rd}}+\boldsymbol{z}_{\mathrm{rd}},
$$

where $\boldsymbol{h}_{r d}=\left[\begin{array}{ll}h_{r_{1} d}[2 k] & h_{r_{2} d}[2 k]\end{array}\right]^{T}$ is the Rayleigh channel coefficient vector and $\boldsymbol{z}_{\mathrm{rd}}=\left[\begin{array}{ll}z_{\mathrm{rd}}[2 k] & z_{\mathrm{rd}}[2 k+1\end{array}\right]^{T}$ is the AWGN noise vector.

At the destination, the Alamouti receiver is used to process the signals received from $R_{1}$ and $R_{2}$. Then, the output of the Alamouti receiver is combined with $\left[y_{\text {sd }}[2 k] \quad y_{\text {sd }}[2 k+1]\right]^{T}$ using the MRC technique.

In the following sections, the performance of the FDSTC protocol is evaluated and compared with conventional DSTC protocols [11-13] in terms of BER, outage probability, diversity order, and energy efficiency. In fact, DSTC protocols are the same as FDSTC except that there is no data exchange between the two relays. In DSTC, the source firstly transmits its signals to the two relays and the destination. Secondly, the relays encode their received signals into a 'distributed' Alamouti code with (NR-relays) or without decoding the received signals (R-relays). Finally, they transmit the received signals to the destination.

\section{Closed-form expressions of the ASEP of the FDSTC protocol}

\subsection{FDSTC protocol with NR-relays}

In this section, a closed-form ASEP of non-regenerative FDSTC (NR-FDSTC) protocol is derived. The SNR at terminal $j\left(j \in\left\{r_{1}, r_{2}, d\right\}\right)$ after receiving signals from terminal $i\left(i \in\left\{s, r_{1}, r_{2}\right\}\right)$ is found as $\gamma_{i j}=\frac{\epsilon_{i j}\left|h_{i j}\right|^{2}}{N_{i j}}$ which has an exponential distribution with the mean $\overline{\gamma_{i j}}=\frac{\epsilon_{i j} \Omega_{i j}}{N_{i j}}$. From (3), the post-detection SNR at the relay $j$ can be referred as

$$
\gamma_{j}=\gamma_{s j}+\frac{\gamma_{s i} \gamma_{i j}}{\gamma_{s i}+\gamma_{i j}+1} \quad i, j \in\left\{r_{1}, r_{2}\right\}, i \neq j .
$$

In the NR-FDSTC protocol, after receiving signals from both the source and the other relay, each relay re-encodes the combined signals $\boldsymbol{u}_{\boldsymbol{j}}$ (3) with Alamouti space-time code to form the Alamouti re-encoded signals $U=$ $\left[\begin{array}{cc}G_{r_{1} d} u_{r_{1}}[2 k] & G_{r_{2} d} u_{r_{2}}[2 k+1] \\ -G_{r_{1} d}^{*} u_{r_{1}}^{*}[2 k+1] & G_{r_{2} d}^{*} u_{r_{2}}^{*}[2 k]\end{array}\right]$.

The combined signals at the relays (3) can be remodeled as

$$
\boldsymbol{u}_{j}=\sqrt{\gamma_{j}} \boldsymbol{x}+\boldsymbol{z}_{u_{j}}, \quad j \in\left\{r_{1}, r_{2}\right\},
$$

where $\gamma_{j}$ is found in (5) and $\boldsymbol{z}_{u_{j}}=\left[z_{u_{j}}[2 k] z_{u_{j}}[2 k+1]\right]^{T}$ is AWGN with zero mean and variance $N_{u_{j}}=1$. To prevent



$G_{j d}=\sqrt{\frac{\epsilon_{j d}}{\gamma_{j}+1}}, \quad j \in\left\{r_{1}, r_{2}\right\}$ with $\epsilon_{j d}$ the transmit power of relay $j$ to the destination $\left(\epsilon_{r}=\epsilon_{r_{1} d}=\epsilon_{r_{2}} d\right)$.

The output signal from the Alamouti receiver is then

$$
\boldsymbol{y}_{r}=\left[\left|h_{r_{1} d} G_{r_{1} d} \sqrt{\gamma_{r_{1}}}\right|^{2}+\left|h_{r_{2} d} G_{r_{2} d} \sqrt{\gamma_{r_{2}}}\right|^{2}\right] \boldsymbol{x}+\boldsymbol{z}_{y_{r}},
$$

where $\boldsymbol{y}_{r}=\left[\begin{array}{ll}y_{r}[2 k] & y_{r}[2 k+1\end{array}\right]^{T}$ and $\boldsymbol{z}_{y_{r}}=\left[\begin{array}{ll}z_{y_{r}}[2 k] & z_{y_{r}}\end{array}\right.$ $[2 k+1]]^{T}$ is AWGN with variance $N_{z_{y_{r}}}=\left[\mid h_{r_{1} d} G_{r_{1} d}\right.$ $\left.\left.\sqrt{\gamma_{r_{1}}}\right|^{2}+\left|h_{r_{2} d} G_{r_{2} d} \sqrt{\gamma_{r_{2}}}\right|^{2}\right]\left[\left|h_{r_{1} d} G_{r_{1} d}\right|^{2}+\left|h_{r_{2} d} G_{r_{2} d}\right|^{2}+1\right]$.

Combining the output signals from Alamouti receiver (7) and the received signals from the source (1) using MRC technique, the signal at the destination is as follows:

$$
\boldsymbol{y}_{d}=\frac{h_{\mathrm{sd}}^{*} \sqrt{\epsilon_{\mathrm{sd}}}}{N_{\mathrm{sd}}} \boldsymbol{y}_{\mathrm{sd}}+\frac{1}{\left|h_{r_{1} d} G_{r_{1} d}\right|^{2}+\left|h_{r_{2} d} G_{r_{2} d}\right|^{2}+1} \boldsymbol{y}_{r},
$$

with $\boldsymbol{y}_{d}=\left[y_{d}[2 k] \quad y_{d}[2 k+1]\right]^{T}$.

Based on (8), the post-detection SNR at the destination can be derived as

$$
\gamma_{d}=\gamma_{\mathrm{sd}}+\frac{\sum_{j \in\left\{r_{1}, r_{2}\right\}} \frac{\gamma_{j d} \gamma_{j}}{\frac{\gamma_{j}+1}{j}}}{\sum_{j \in\left\{r_{1}, r_{2}\right\}} \frac{\gamma_{j d}}{\gamma_{j}+1}+1} .
$$

In this part, a closed-form ASEP of the NR-FDSTC protocol is derived when the relay-relay SNR is much larger than the source-relay SNR $\left(\gamma_{i j} \gg \gamma_{s i}\right)$. One should note that the assumption can be practically obtained in dense WSNs even with $\epsilon_{\mathrm{rr}}=1 / 10 \epsilon_{s}$. Equation 5 can be rewritten as $\gamma_{j} \approx \gamma_{s j}+\gamma_{s i} \quad i, j \in\left\{r_{1}, r_{2}\right\}, i \neq j$. One should note that the equality happens $\left(\gamma_{j}=\gamma_{s j}+\gamma_{s i}\right)$ when there is no error in the data exchange between the two relays.

Since $\gamma_{r_{1}}=\gamma_{r_{2}}=\gamma_{r} \approx \gamma_{\mathrm{sr}_{1}}+\gamma_{\mathrm{sr}_{2}}$, (9) can be approximated by

$$
\begin{aligned}
\gamma_{d} \approx \gamma_{\mathrm{sd}}+\frac{\left(\gamma_{r_{1} d}+\gamma_{r_{2} d}\right) \gamma_{r}}{\gamma_{r_{1} d}+\gamma_{r_{2} d}+\gamma_{r}+1} & =\gamma_{\mathrm{sd}}+\frac{\gamma_{\mathrm{rd}} \gamma_{r}}{\gamma_{\mathrm{rd}}+\gamma_{r}+1} \\
& =\gamma_{\mathrm{sd}}+\gamma_{\mathrm{srd}}, \quad \text { (10) }
\end{aligned}
$$

with $\gamma_{\mathrm{rd}}=\gamma_{r_{1} d}+\gamma_{r_{2} d}$ and $\gamma_{\mathrm{srd}}=\frac{\gamma_{\mathrm{rd}} \gamma_{r}}{\gamma_{\mathrm{rd}}+\gamma_{r}+1}$.

Once again, using the moment generating function (MGF)-based approach, the closed form ASEP of the NRFDSTC protocol can be derived by evaluating the MGF of $\gamma_{\mathrm{sd}}$ and $\gamma_{\text {srd }}$. The MGF of $\gamma_{\mathrm{sd}}$ is already shown in [9].

The MGF of $\gamma_{\text {srd }}$, proved in Appendix 1, is given by

$$
\begin{aligned}
M_{\gamma_{\text {srd }}}(s)= & e^{\alpha / 2}\left[\frac { 2 } { p \sqrt { p } } \left(-\left(1-\frac{\sigma^{2}}{p}\right) \frac{\delta J_{0}}{\delta \beta}+\left(\frac{\sigma^{2}}{p}-\frac{2 \sigma}{p}-2\right)\right.\right. \\
& \left.\times \frac{\delta^{2} J_{0}}{\delta \alpha \delta \beta}-\frac{4 \sigma}{p} \frac{\delta^{3} J_{0}}{\delta \beta \delta \alpha^{2}}\right) \\
+ & \frac{2}{p^{2}}\left(-\left(\frac{\sigma^{2}}{p}+4\right) \frac{\delta^{3} J_{0}}{\delta \alpha^{3}}-\frac{1}{2}\left(\frac{\sigma^{2}}{p}+4\right) \frac{\delta^{2} J_{0}}{\delta \alpha^{2}}\right. \\
& \left.\left.+\frac{\sigma^{2}}{p} \frac{\delta J_{0}}{\delta \alpha}-\frac{\sigma^{2}}{8 p} J_{0}\right)\right],
\end{aligned}
$$


where $\sigma=\overline{\gamma_{\text {rd }}}+\overline{\gamma_{r}}, p=\overline{\gamma_{r d} \gamma_{r}}$, and $\alpha=(\sigma-p s) / p$. The formulas of $J_{0}, \frac{\delta J_{0}}{\delta \alpha}, \frac{\delta J_{0}}{\delta \beta}, \frac{\delta^{2} J_{0}}{\delta \alpha^{2}}, \frac{\delta^{2} J_{0}}{\delta \alpha \delta \beta}, \frac{\delta^{3} J_{0}}{\delta \beta \delta \alpha^{2}}$ and $\frac{\delta^{3} J_{0}}{\delta \alpha^{3}}$ can be found in Appendix 1. Note that the random variable $\gamma_{\mathrm{rd}}=\gamma_{r_{1} d}+\gamma_{r_{2} d}$ will have a probability density function $f_{\gamma_{\mathrm{rd}}}(\gamma)=\frac{1}{\bar{\gamma}_{\mathrm{rd}}} \gamma e^{-\frac{\gamma}{\gamma_{\mathrm{rd}}}}$. Similarly, the probability density function of random variable $\gamma_{r}=\gamma_{\mathrm{sr}_{1}}+\gamma_{\mathrm{sr}_{2}}$ is $f_{\gamma_{r}}(\gamma)=$ $\frac{1}{\overline{\gamma r}^{2}} \gamma e^{-\frac{\gamma}{\gamma_{r}}}$.

The ASEP of the NR-FDSTC protocol with BPSK modulation can be derived as

$$
P_{\gamma_{d}}=\frac{1}{\pi} \int_{0}^{\pi / 2} \frac{M_{\gamma_{\mathrm{srd}}}\left(-\frac{g_{\mathrm{BPSK}}}{\sin ^{2} \theta}\right)}{1+\frac{g_{\mathrm{BSSK}} \overline{\gamma_{\mathrm{sd}}}}{\sin ^{2} \theta}} d \theta,
$$

where $g_{\mathrm{BPSK}}=1[27]$.

\subsection{FDSTC protocol with R-relays}

In this work, the destination is not aware of the error probabilities at the relays, making the use of the maximum likelihood (ML) decoder at the destination impossible. Therefore, taking the decoding errors at the two relays into account, the error probability of regenerative FDSTC (R-FDSTC) protocol conditioned to the channel, is derived via a sub-optimal decoder at the destination. The Alamouti re-encoded signals $U$ of the R-FDSTC protocol have the form of

$$
U=\left[\begin{array}{cc}
\sqrt{\epsilon_{r_{1}}} \hat{x}_{r_{1}}[2 k] & \sqrt{\epsilon_{r_{2}}} \hat{x}_{r_{2}}[2 k+1] \\
-\sqrt{\epsilon_{r_{1}}} \hat{x}_{r_{1}}^{*}[2 k+1] & \sqrt{\epsilon_{r_{2}}} \hat{x}_{r_{2}}^{*}[2 k]
\end{array}\right]
$$

where $\hat{x}_{j}[n]$ is obtained by decoding the combined signal $u_{j}[n]$ (3) at the relay $j \in\left\{r_{1}, r_{2}\right\}$ using ML decoder.

Using the MRC technique, the combined signal at the destination is as follows:

$$
\begin{aligned}
y_{d}[2 k]= & \frac{\epsilon_{s}\left|h_{\mathrm{sd}}\right|^{2}}{N_{\mathrm{sd}}} x[2 k]+\frac{\epsilon_{r_{1}}\left|h_{r_{1} d}\right|^{2}}{N_{r_{1} d}} \hat{x}_{r_{1}}[2 k]+\frac{\epsilon_{r_{2}}\left|h_{r_{2} d}\right|^{2}}{N_{r_{2} d}} \hat{x}_{r_{2}}[2 k] \\
& -\frac{\sqrt{\epsilon_{r_{1}} \epsilon_{r_{2}}} h_{r_{1} d}^{*} h_{r_{2} d} d}{N_{r_{2} d}} \hat{x}_{r_{1}}[2 k+1] \\
& +\frac{\sqrt{\epsilon_{r_{1}} \epsilon_{r_{2}}} h_{r_{1} d}^{*} h_{r_{2} d}}{N_{r_{1} d}} \hat{x}_{r_{2}}[2 k+1]+z_{y_{d}}[2 k],
\end{aligned}
$$

where $z_{y_{d}}$ is AWGN with zero mean and variance $N_{z_{y_{d}}}=$ $\frac{\epsilon_{r_{1}}\left|h_{r_{1}}\right|^{2}}{N_{r_{1} d}}+\frac{\epsilon_{r_{2}}\left|h_{r_{2} d}\right|^{2}}{N_{r_{2} d}}+\frac{\epsilon_{s}\left|h_{s d}\right|^{2}}{N_{s d}}$.

The sub-optimal scalar decoder [14] is used instead of the ML decoder to decode the combined signal $y_{d}[2 k]$. The decision on the transmitted symbol $x[2 k]$ can be specifically obtained as

$$
\hat{x}[2 k]=\operatorname{sign}\left\{\operatorname{Re}\left\{y_{d}[2 k]\right\}\right\} .
$$

Then, in the presence of the decision errors at the relays, the closed-form expression of the error probability, conditioned to the channel, for the R-FDSTC protocol can be derived as (see Appendix 2 for more details)

$$
\begin{aligned}
P_{2 k}= & \frac{1}{2}\left(1-p_{2 k}^{r_{1}}\right)\left(1-p_{2 k}^{r_{2}}\right)\left[\left(1-p_{2 k+1}^{r_{1}}\right)\left(1-p_{2 k+1}^{r_{2}}\right)\right. \\
& \left.+p_{2 k+1}^{r_{1}} p_{2 k+1}^{r_{2}}\right] \operatorname{erfc}\left(\beta_{2 k}\right) \\
& +\frac{1}{2}\left(1-p_{2 k}^{r_{1}}\right) p_{2 k}^{r_{2}}\left[\left(1-p_{2 k+1}^{r_{1}}\right)\left(1-p_{2 k+1}^{r_{2}}\right)\right. \\
& \left.+p_{2 k+1}^{r_{1}} p_{2 k+1}^{r_{2}}\right] \operatorname{erfc}\left(\delta_{2 k}\right) \\
& +\frac{1}{2} p_{2 k}^{r_{1}}\left(1-p_{2 k}^{r_{2}}\right)\left[\left(1-p_{2 k+1}^{r_{1}}\right)\left(1-p_{2 k+1}^{r_{2}}\right)\right. \\
& \left.+p_{2 k+1}^{r_{1}} p_{2 k+1}^{r_{2}}\right] \operatorname{erfc}\left(\lambda_{2 k}\right) \\
& +\frac{1}{2} p_{2 k}^{r_{1}} p_{2 k}^{r_{2}}\left[\left(1-p_{2 k+1}^{r_{1}}\right)\left(1-p_{2 k+1}^{r_{2}}\right)+p_{2 k+1}^{r_{1}} p_{2 k+1}^{r_{2}}\right] \\
& \times \operatorname{erfc}\left(\phi_{2 k}\right) \\
& +\frac{1}{4}\left(1-p_{2 k}^{r_{1}}\right)\left(1-p_{2 k}^{r_{2}}\right)\left[p_{2 k+1}^{r_{1}}\left(1-p_{2 k+1}^{r_{2}}\right)\right. \\
& \left.+\left(1-p_{2 k+1}^{r_{1}}\right) p_{2 k+1}^{r_{2}}\right]\left[\operatorname{erfc}\left(\beta_{2 k}+\theta_{2 k}\right)+\operatorname{erfc}\left(\beta_{2 k}-\theta_{2 k}\right)\right] \\
& +\frac{1}{4}\left(1-p_{2 k}^{r_{1}}\right) p_{2 k}^{r_{2}}\left[p_{2 k+1}^{r_{1}}\left(1-p_{2 k+1}^{r_{2}}\right)+\left(1-p_{2 k+1}^{r_{1}}\right) p_{2 k+1}^{r_{2}}\right] \\
& \times\left[\operatorname{erfc}\left(\delta_{2 k}+\theta_{2 k}\right)+\operatorname{erfc}\left(\delta_{2 k}-\theta_{2 k}\right)\right] \\
& +\frac{1}{4} p_{2 k}^{r_{1}}\left(1-p_{2 k}^{r_{2}}\right)\left[p_{2 k+1}^{r_{1}}\left(1-p_{2 k+1}^{r_{2}}\right)+\left(1-p_{2 k+1}^{r_{1}}\right) p_{2 k+1}^{r_{2}}\right] \\
& \times\left[\operatorname{erfc}\left(\lambda_{2 k}+\theta_{2 k}\right)+\operatorname{erfc}\left(\lambda_{2 k}-\theta_{2 k}\right)\right] \\
& +\frac{1}{4} p_{2 k}^{r_{1}} p_{2 k}^{r_{2}}\left[p_{2 k+1}^{r_{1}}\left(1-p_{2 k+1}^{r_{2}}\right)+\left(1-p_{2 k+1}^{r_{1}}\right) p_{2 k+1}^{r_{2}}\right] \\
& \times\left[\operatorname{erfc}\left(\phi_{2 k}+\theta_{2 k}\right)+\operatorname{erfc}\left(\phi_{2 k}-\theta_{2 k}\right)\right] \\
& {[16) }
\end{aligned}
$$

where

$$
\begin{aligned}
\beta_{2 k} & =\sqrt{\gamma_{r_{1} d}+\gamma_{r_{2} d}+\gamma_{\mathrm{sd}}} ; \quad \delta_{2 k}=\frac{\gamma_{r_{1} d}-\gamma_{r_{2} d}+\gamma_{\mathrm{sd}}}{\sqrt{\gamma_{r_{1} d}+\gamma_{r_{2} d}+\gamma_{\mathrm{sd}}}} \\
\lambda_{2 k} & =\frac{-\gamma_{r_{1} d}+\gamma_{r_{2} d}+\gamma_{\mathrm{sd}}}{\sqrt{\gamma_{r_{1} d}+\gamma_{r_{2} d}+\gamma_{\mathrm{sd}}}} ; \quad \phi_{2 k}=\frac{-\gamma_{r_{1} d}-\gamma_{r_{2} d}+\gamma_{\mathrm{sd}}}{\sqrt{\gamma_{r_{1} d}+\gamma_{r_{2} d}+\gamma_{\mathrm{sd}}}} \\
\theta_{2 k} & =\frac{\sqrt{\epsilon_{r_{1}} \epsilon_{r_{2}} \operatorname{Re}\left\{h_{r_{1} d}^{*} h_{r_{2} d}\right\}}}{\sqrt{\gamma_{r_{1} d}+\gamma_{r_{2} d}+\gamma_{\mathrm{sd}}}}\left(\frac{1}{N_{r_{1} d}}+\frac{1}{N_{r_{2} d}}\right) ; \\
\operatorname{erfc}(z) & =\int_{z}^{\infty} \frac{\exp \left(-x^{2} / 2\right)}{\sqrt{2 \pi}} d x
\end{aligned}
$$

and $p_{2 k}^{j}, p_{2 k+1}^{j}\left(j \in\left\{r_{1}, r_{2}\right\}\right)$ are the conditional error probabilities at relay $j$ on $x[2 k]$ and $x[2 k+1]$, respectively. When considering the R-DSTC protocol, the R-FDSTC protocol without transmission errors between relays (ideal case) and the R-FDSTC protocol with transmission errors between relays, $p_{2 k}^{j}$ can be found as 


$$
p_{2 k}^{j}= \begin{cases}\frac{1}{2} \operatorname{erfc}\left(\sqrt{\gamma_{s j}}\right), & \text { R-DSTC protocol } \\ \frac{1}{2} \operatorname{erfc}\left(\sqrt{\gamma_{\mathrm{sr}_{1}}+\gamma_{\mathrm{sr}_{2}}}\right), & \text { R-FDSTC protocol without transmission errors between relays } \\ \frac{1}{2} \operatorname{erfc}\left(\sqrt{\gamma_{s j}+\frac{\gamma_{s i} \gamma_{i j}}{\gamma_{s i}+\gamma_{i j}+1}}\right), & \text { R-FDSTC protocol with transmission errors between relays }\end{cases}
$$

\section{Outage probability}

In this section, the DSTC and FDSTC protocols are considered in terms of outage probability while having the same spectral efficiency $R(\mathrm{bits} / \mathrm{s} / \mathrm{Hz})$. Using the methodology in [1] to find the approximations of the outage probability of the protocols, it is shown that the NR-FDSTC protocol can achieve the maximum diversity order despite using AGC at the relays. Besides, the R-FDSTC protocol also brings us a higher diversity oder in comparison with the R-DSTC protocol.

\subsection{Outage probability analysis}

Outage probability is defined as $P_{\text {out }}=\operatorname{Pr}\{I<R\}$ where $I$ is the maximum average mutual information between source and destination and $R$ is the spectral efficiency. In this work, without loss of generality we note $\Omega_{\text {sr }}=$ $\Omega_{\mathrm{sr}_{1}}=\Omega_{\mathrm{sr}_{2}}$ and $\Omega_{\mathrm{rd}}=\Omega_{r_{1} d}=\Omega_{r_{2} d}$. The function $f(x, y)=\frac{x y}{x+y+1}$ used for the fading coefficients in the following expressions is defined in [1].

For a fair comparison, once again, the total transmit power is the same for the two protocols, i.e., $\epsilon_{s}+2 \epsilon_{r}=P$ (DSTC) and $\epsilon_{s}+2 \epsilon_{r}+2 \epsilon_{\mathrm{rr}}=P\left(\right.$ FDSTC with $\left.\epsilon_{\mathrm{rr}}=\frac{1}{10} \epsilon_{r}\right)$. Let the transmit power be equally allocated among source and relays $\left(\epsilon_{s}=\epsilon_{r}=\epsilon, N_{i j}=N_{0}(\forall i, j)\right.$ and SNR $=$ $\epsilon / N_{0}$. The outage probability of the protocols is determined based on the SNR parameter for different types of relays. For shortening the following expressions, we note $\left|h_{\mathrm{sr}}\right|^{2}=\min \left\{\left|h_{\mathrm{sr}_{1}}\right|^{2},\left|h_{\mathrm{sr}_{2}}\right|^{2}\right\},\left.|| \boldsymbol{a}_{\mathrm{sr}}\right|^{2}=\left|h_{\mathrm{sr}_{1}}\right|^{2}+\left|h_{\mathrm{sr}_{2}}\right|^{2}$, and $\left\|\boldsymbol{a}_{\mathrm{rd}}\right\|^{2}=\left|h_{r_{1} d}\right|^{2}+\left|h_{r_{2} d}\right|^{2}$.

\subsubsection{NR-relays}

a/ DSTC protocol The maximum average mutual information can be expressed as

$$
I_{\mathrm{DSTC}}^{\mathrm{NR}}=\frac{1}{2} \log \left(1+\mathrm{SNR} \cdot\left|h_{\mathrm{sd}}\right|^{2}+f\left(\mathrm{SNR} \cdot\left|h_{\mathrm{sr}}\right|^{2},\left.\mathrm{SNR} \cdot|| \boldsymbol{a}_{\mathrm{rd}}\right|^{2}\right)\right) .
$$

In the high SNR region, the outage probability of the DSTC protocol can be approximated as

$$
\begin{aligned}
\operatorname{Pr}\left\{I_{\mathrm{DSTC}}^{\mathrm{NR}}<R\right\} & =\operatorname{Pr}\left\{\left|h_{\mathrm{sd}}\right|^{2}+\frac{1}{\mathrm{SNR}} f\left(\mathrm{SNR} \cdot\left|h_{\mathrm{sr}}\right|^{2}, \mathrm{SNR} \cdot|| \boldsymbol{a}_{\mathrm{rd}} \|^{2}\right)\right. \\
& \left.<\frac{2^{2 R}-1}{\mathrm{SNR}}\right\} \\
& \sim \frac{1}{2} \frac{1}{\Omega_{\mathrm{sr}}} \frac{1}{\Omega_{\mathrm{sd}}}\left(\frac{2^{2 R}-1}{\mathrm{SNR}}\right)^{2} \text { when SNR } \rightarrow \infty .
\end{aligned}
$$

Using AGC at the relays, it is claimed in [12] that the NR-DSTC protocol can not achieve full diversity. Here, the maximum diversity order is only two. This result is totally suitable to the simulation results in [12].

b/ FDSTC protocol The maximum average mutual information can be derived as

$$
\begin{aligned}
I_{\mathrm{FDSTC}}^{\mathrm{NR}}= & \frac{1}{4} \log \left(1+\mathrm{SNR} \cdot\left|h_{\mathrm{sd}}\right|^{2}\right. \\
& \left.+f\left(\mathrm{SNR} \cdot\left\|\boldsymbol{a}_{\mathrm{sr}}\right\|^{2}, \mathrm{SNR} \cdot\left\|\boldsymbol{a}_{\mathrm{rd}}\right\|^{2}\right)\right) .
\end{aligned}
$$

The approximation of the outage probability in the high SNR region is

$$
\begin{aligned}
\operatorname{Pr}\left\{I_{\mathrm{FDSTC}}^{\mathrm{NR}}<R\right\} & =\operatorname{Pr}\left\{\left|h_{\mathrm{sd}}\right|^{2}+\frac{1}{\mathrm{SNR}} f\left(\mathrm{SNR} \cdot\left\|\boldsymbol{a}_{\mathrm{sr}}\right\|^{2}, \mathrm{SNR} \cdot\left\|\boldsymbol{a}_{\mathrm{rd}}\right\|^{2}\right)\right. \\
& \left.<\frac{2^{4 R}-1}{\mathrm{SNR}}\right\} \\
& \sim \frac{1}{3 !} \frac{\Omega_{\mathrm{sr}}^{2}+\Omega_{\mathrm{rd}}^{2}}{\Omega_{\mathrm{sr}}^{2} \Omega_{\mathrm{rd}}^{2}} \frac{1}{\Omega_{\mathrm{sd}}}\left(\frac{2^{4 R}-1}{\mathrm{SNR}}\right)^{3} \text { when SNR } \rightarrow \infty
\end{aligned}
$$

With NR-relays, the FDSTC protocol can achieve full diversity order.

\subsubsection{R-relays}

a/ DSTC protocol The maximum average mutual information can be expressed as

$$
\begin{aligned}
I_{\mathrm{DSTC}}^{R}= & \frac{1}{2} \min \left\{\log \left(1+\mathrm{SNR} \cdot\left|h_{\mathrm{sr}}\right|^{2}\right),\right. \\
& \left.\log \left(1+\operatorname{SNR} .\left(\left|h_{\mathrm{sd}}\right|^{2}+\left.|| \boldsymbol{a}_{\mathrm{rd}}\right|^{2}\right)\right)\right\} .
\end{aligned}
$$

The outage probability in high SNR regions can be approximated as

$$
\begin{aligned}
\operatorname{Pr}\left\{I_{\mathrm{DSTC}}^{R}<R\right\} & =\operatorname{Pr}\left\{\min \left\{\left|h_{\mathrm{sr}}\right|^{2},\left|h_{\mathrm{sd}}\right|^{2}+\left.|| \boldsymbol{a}_{\mathrm{rd}}\right|^{2}\right\}<\frac{2^{2 R}-1}{\mathrm{SNR}}\right\} \\
& \sim \frac{1}{\Omega_{\mathrm{sr}}}\left(\frac{2^{2 R}-1}{\mathrm{SNR}}\right) \text { when SNR } \rightarrow \infty .
\end{aligned}
$$

The diversity order of the R-DSTC system is only 1.

b/ FDSTC protocol The maximum average mutual information can be derived as

$$
\begin{aligned}
I_{\mathrm{FDSTC}}^{R}= & \frac{1}{4} \min \left\{\log \left(1+\mathrm{SNR} \cdot\left\|\boldsymbol{a}_{\mathrm{sr}}\right\|^{2}\right),\right. \\
& \left.\log \left(1+\mathrm{SNR} .\left(\left|h_{\mathrm{sd}}\right|^{2}+\left\|\boldsymbol{a}_{\mathrm{rd}}\right\|^{2}\right)\right)\right\} .
\end{aligned}
$$


In high SNR regions, the outage probability becomes

$$
\begin{aligned}
\operatorname{Pr}\left\{I_{\mathrm{FDSTC}}^{R}<R\right\} & =\operatorname{Pr}\left\{\min \left\{\left\|\boldsymbol{a}_{\mathrm{sr}}\right\|^{2},\left|h_{\mathrm{sd}}\right|^{2}+\left\|\boldsymbol{a}_{\mathrm{rd}}\right\|^{2}\right\}<\frac{2^{4 R}-1}{\mathrm{SNR}}\right\} \\
& \sim \frac{1}{2} \frac{1}{\Omega_{\mathrm{sr}}^{2}}\left(\frac{2^{4 R}-1}{\mathrm{SNR}}\right)^{2} \text { when SNR } \rightarrow \infty .
\end{aligned}
$$

The R-FDSTC protocol can not achieve the full diversity. However, it gets higher system diversity order in comparison with the R-DSTC protocol.

\subsection{Diversity-multiplexing trade-off (DMT)}

The diversity-multiplexing trade-off illuminates the relationship between the reliability of data transmissions in terms of diversity gain and the spectral efficiency in terms of multiplexing gain. The multiplexing gain, which illustrates how fast the source data rate varies with respect to $\mathrm{SNR}$, is defined as [28]

$$
g=\lim _{\mathrm{SNR} \rightarrow \infty} \frac{R}{\log \mathrm{SNR}} .
$$

The diversity gain $d(g)$ can be characterized as a function of $g$ [28]

$$
d(g)=\lim _{\mathrm{SNR} \rightarrow \infty}-\frac{\log P_{\mathrm{out}}}{\log \mathrm{SNR}}
$$

Based on the approximations of outage probability for the protocols in the above parts, the DMT of the DSTC and FDSTC protocols for NR-relays and R-relays can be expressed respectively as

$$
\begin{aligned}
d_{\text {DSTC }}^{\mathrm{NR}}(g) & =2(1-2 g), \\
d_{\mathrm{FDSTC}}^{\mathrm{NR}}(g) & =3(1-4 g), \\
d_{\mathrm{DSTC}}^{R}(g) & =1(1-2 g), \\
d_{\mathrm{FDSTC}}^{R}(g) & =2(1-4 g),
\end{aligned}
$$

\section{Energy efficiency}

To have a fair comparison in the total power consumption of the two protocols, the total energy consumption for both protocols (FDSTC and DSTC) is considered. Obviously, the FDSTC protocol consumes more transmission and circuit energy due to the inter-relay communication. However, by simulations, the FDSTC protocol is shown to be an energy-efficient protocol for long-range transmissions.

The total energy consumption per bit of the FDSTC protocol $E_{\mathrm{FDSTC}}^{b}$ can be calculated by the sum of the total energy consumption per bit of the broadcast phase of the source $E_{s}^{b}$, the data exchange phase between the two relays $E_{\mathrm{rr}}^{b}$, the transmission phase from the relays to the destination $E_{r d}^{b}$. The total energy consumption per bit of the conventional DSTC protocol $E_{\mathrm{DSTC}}^{b}$ is the same except that there is no energy consumption for the data exchange phase between the relays.

$$
\begin{aligned}
E_{\mathrm{FDSTC}}^{b} & =E_{s}^{b}+E_{\mathrm{rr}}^{b}+E_{\mathrm{rd}}^{b}, \\
E_{\mathrm{DSTC}}^{b} & =E_{s}^{b}+E_{\mathrm{rd}}^{b} .
\end{aligned}
$$

Note that $E_{s}^{b}, E_{\mathrm{rr}}^{b}$, and $E_{\mathrm{rd}}^{b}$ are calculated based on the typical energy model proposed in [21].

\section{Simulation results}

Assuming that both relays have the same distance from source $d_{\mathrm{sr}}=d_{\mathrm{sr}_{1}}=d_{\mathrm{sr}_{2}}$, let denote $r=\frac{d_{\mathrm{sr}}}{d_{\mathrm{sd}}}$ the relative distance of relays defined by the ratio of the source-relay distance $d_{\mathrm{sr}}$ and the source-destination distance $d_{\mathrm{sd}}$ and $\mathrm{rr}=\frac{d_{\mathrm{rr}}}{d_{\mathrm{sd}}}$ the relative inter-distance of relays defined by the ratio of the relay-relay distance $d_{\mathrm{rr}}$ and the sourcedestination distance. In this section, the BER performance of the FDSTC protocol is evaluated for NR-relays and Rrelays. Using a common transmission model, we set the path loss $\Omega_{i j} \propto d_{i j}^{-\mathrm{pl}}$ where $d_{i j}$ is the distance between terminal $i$ and terminal $j$ and $\mathrm{pl}$ is the path loss exponent. In our simulations, $\mathrm{pl}=2$ is used and BPSK modulation is considered. For a fair comparison, the total transmit power of the protocol is kept as the same (i.e., $P$ ). For the DSTC protocol $\epsilon_{s}+2 \epsilon_{r}=P$. On the other hand, for the FDSTC protocol, we have $\epsilon_{s}+2 \epsilon_{r}+2 \epsilon_{\mathrm{rr}}=P\left(\epsilon_{\mathrm{rr}}=\frac{1}{10} \epsilon_{r}\right)$. Unless otherwise stated, we assume that $\epsilon_{s}=\epsilon_{r}$.

\subsection{BER performance}

\subsubsection{FDSTC protocol vs. DSTC protocol}

Figure 2 shows the BER comparison between the FDSTC and DSTC protocols as a function of the SNR $\left(P / N_{0}\right)$ under some cases of the relative inter-distance between relays, rr. Figure 2a shows the simulations for NR-relays while the simulations for R-relays are shown in Figure $2 b$. In these simulations, the relays are in the middle of the source and the destination $(r=0.5)$. The performance of the FDSTC protocol in terms of BER is shown to be much better than that of the DSTC protocol, thanks to the data exchange between the relays. When the relative interdistance of relays is small $(\mathrm{rr}<0.2)$, the performance of the FDSTC protocol is still comparable with the ideal case $\left(\Omega_{\mathrm{rr}} \gg \Omega_{\mathrm{sr}}\right)$ where there is no errors in the data exchange between the two relays. However, when the quality of the relay-relay link is poor $\left(\Omega_{\mathrm{rr}} \ll \Omega_{\mathrm{sr}}\right)$, the BER performance of the FDSTC protocol logically tends to that of the DSTC protocol since the inter-relay transmission does not bring any gain as compared to the DSTC protocol. Mathematically, when $\frac{\Omega_{\mathrm{rr}}}{\Omega_{\mathrm{sr}}} \rightarrow 0$, the post-destination SNR at relays $\gamma_{j}$ (5) tends to the source-relay SNR $\gamma_{s j}\left(\gamma_{j} \approx \gamma_{s j}+\gamma_{i j} \approx \gamma_{s j}\right)$ as a result of $\gamma_{s j}, \gamma_{s i} \gg \gamma_{i j}$. That leads to equal BER performance between DSTC and FDSTC. 


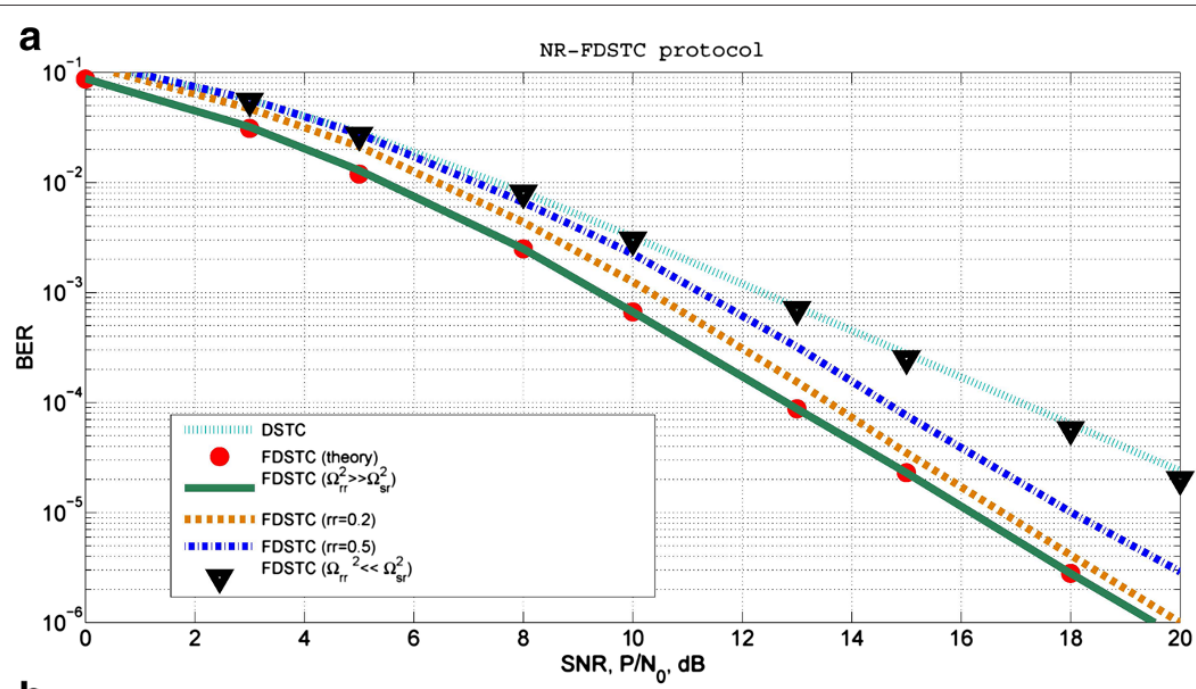

b

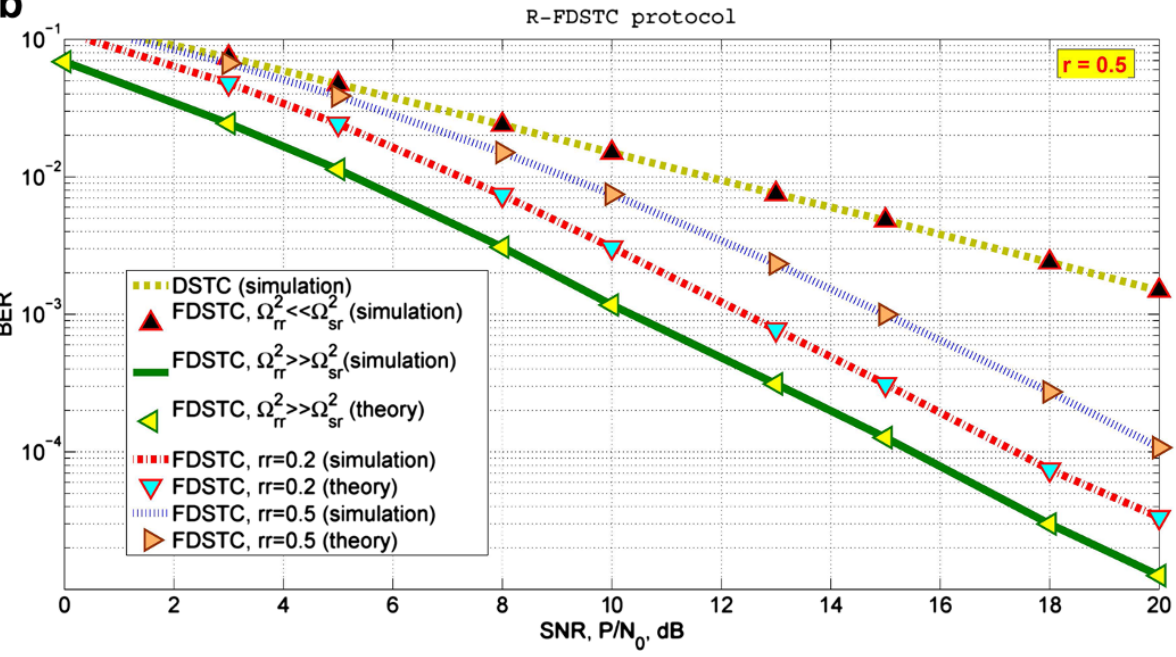

Figure 2 Impact of the inter-distance between relays when the relays are in the middle of the source and the destination $(r=0.5)$. For (a) NR-FDSTC protocol and (b) R-FDSTC protocol.

\subsubsection{Impact of the relative distance of relays}

The SNR gain of the FDSTC protocol over the conventional DSTC protocol versus the relative distance of relays at a desired BER $=10^{-5}$ is shown in Figure 3 . The average SNR gain of the NR-FDSTC protocol over the NR-DSTC protocol is $3.7 \mathrm{~dB}$. However, the average SNR gain of the R-FDSTC protocol over the R-DSTC protocol is even much more impressive. By using the simple data exchange between the two relays, we can get an average SNR gain up to $18.1 \mathrm{~dB}$.

The impact of the relative distance of relays on the required SNR of the FDSTC and DSTC protocols is shown in Figure 4. At a desired BER $=10^{-5}$, the required SNR is shown as a function of the relative distance of relays. Obviously, there will be a difference in the required SNR of each protocol when the relative distance of relays, $r$ changes from 0.1 to 0.9 .
For the NR-DSTC protocol, the difference is $6.3 \mathrm{~dB}$. However, for the NR-FDSTC protocol, this difference is decreased to $1.6 \mathrm{~dB}$, which means that the impact of the relative distance of relays is reduced by up to $75 \%$. For Rrelays, we still get up to a $64 \%$ reduction of the impact of the relative distance of relays. The difference of required SNR changes from $20.5 \mathrm{~dB}$ in case of the R-DSTC protocol down to $7.4 \mathrm{~dB}$ in case of the R-FDSTC protocol. This is a very interesting result especially for the mobile relays since for the FDSTC protocol, the required SNR has almost no change (NR-relays) or a little change (R-relays) when the relays are moving between the source and the destination.

\subsubsection{Impact of synchronization errors}

Like cooperative MIMO, distributed space-time coded relays suffer from the impact of synchronization errors 


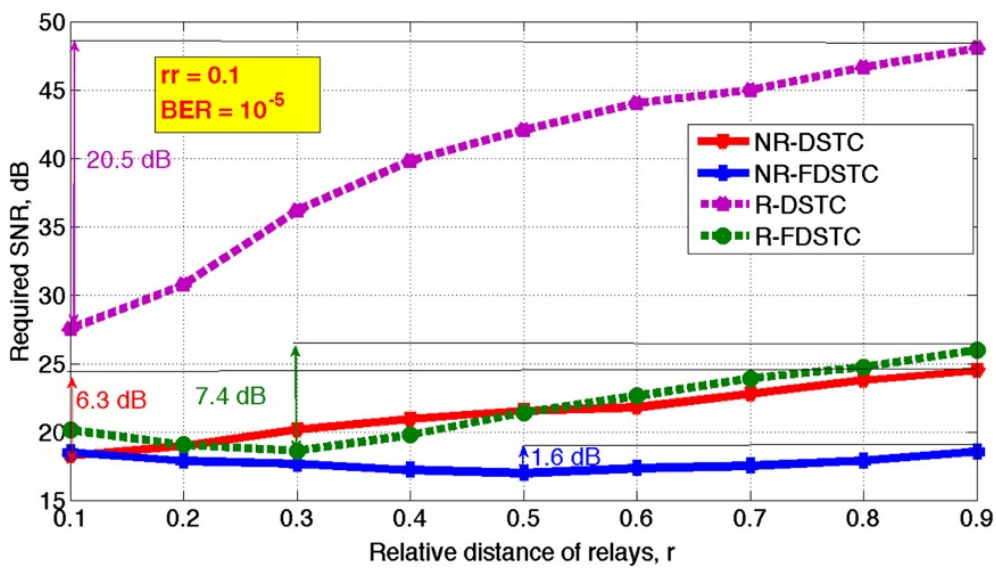

Figure 3 SNR gain of the FDSTC protocol over the conventional DSTC protocol. As a function of the relative distance of relays $\left(B E R=10^{-5}\right.$ and $r \mathrm{r}=0.1$ ).

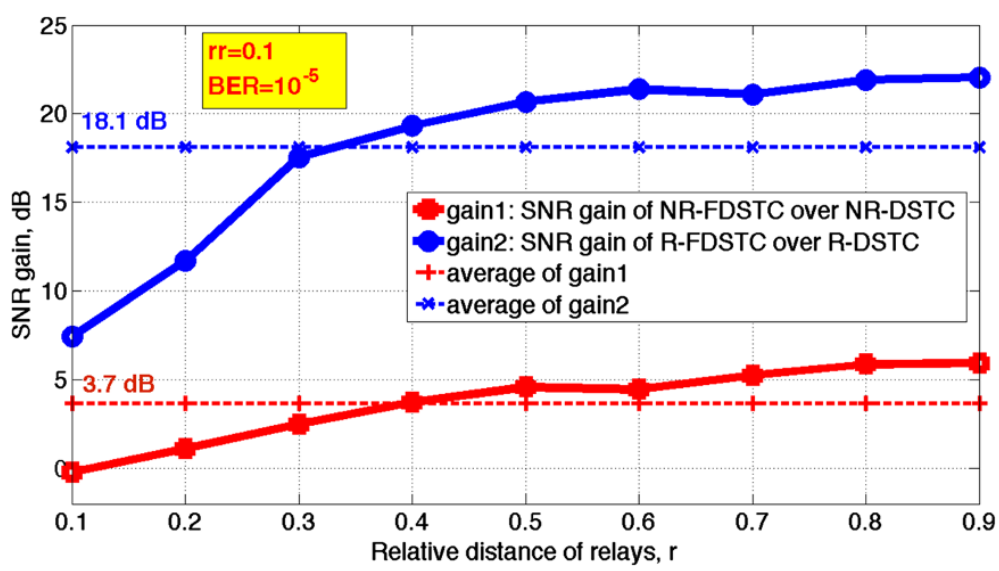

Figure 4 Required SNR versus relative distance of relays. For a desired $B E R=10^{-5}$ and when the relative inter-distance of relays is equal to 0.1 $(r r=0.1)$.

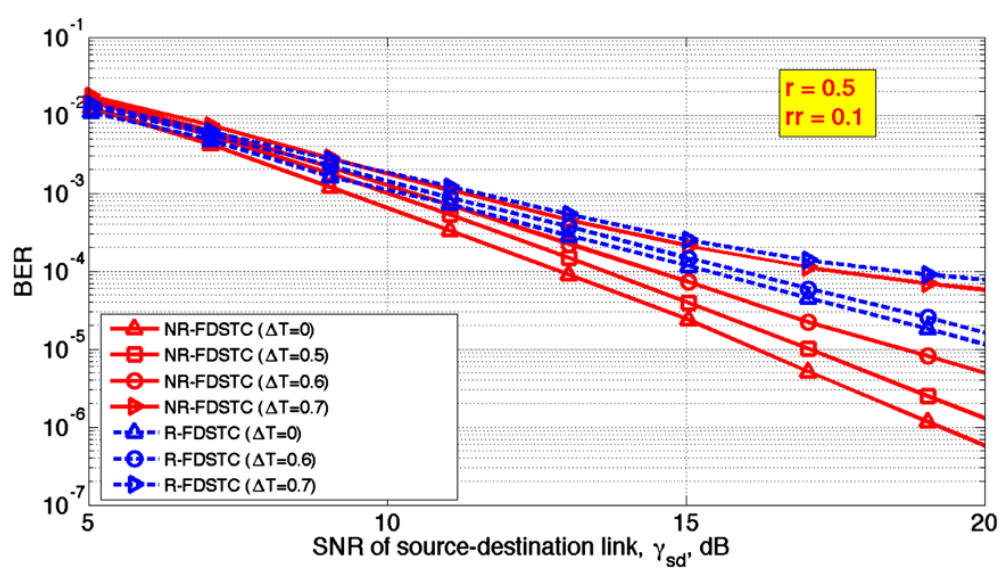

Figure 5 Impact of synchronization errors of the relay-destination link for different protocols ( $r r=0.1$ and $r=0.5$ ). 
[29]. In all above simulations, we supposed that the transmission from the relays to the destination is perfectly synchronized, i.e., simultaneous between the relays, which cannot happen in real systems. In our model, as a consequence of using DSTC relays in the FDSTC protocol, the synchronization errors have large impacts on the performance of the relay-destination link and therefore reduce the performance of the overall system. Figure 5 shows the impacts of synchronization errors on the overall performance of our model for two protocols: R-FDSTC and NR-FDSTC when $\mathrm{rr}=0.1$ and $r=0.5$. The synchronization error model of [30] where the interference inter-symbol (ISI) is only created by the four nearest symbols is considered. In our simulations, the synchronization error is considered to have a uniform distribution in the range $[-\Delta T / 2, \Delta T / 2]$, with $\Delta T$ the synchronization error range normalized by the symbol period. The overall performance of the system is still acceptable when $\Delta T<0.6$ for R-FDSTC or $\Delta T<0.5$ for NRFDSTC. The performance becomes saturated when $\Delta T=$ 0.7. Synchronization methods for these protocols are beyond the scope of this paper and may be considered as future work.

\subsubsection{Power allocation}

Let $\epsilon_{s}=c_{P} P\left(0<c_{P}<1\right)$ and $\epsilon_{r}=\frac{10}{22}\left(1-c_{P}\right) P$ (note that $\left.\epsilon_{\mathrm{rr}}=\frac{1}{10} \epsilon_{r}\right)$. Using an exhaustive search by simulation, we can find the best value of $c_{P}$ (i.e., $c_{P}^{\text {opt }}$ ) to further increase the BER performance of the FDSTC protocol as shown in Figure 6. The best power allocation points of the FDSTC protocol for NR-relays and R-relays are respectively $c_{P}^{\text {opt }}=$ 0.4 and $c_{P}^{\text {opt }}=0.6$ when considered in the case BER $=$ $10^{-5}, r=0.5$, and $\mathrm{rr}=0.1$. In addition, as compared to the case of equal power allocation (i.e., $c_{P}=10 / 32$ ), SNR gains of $0.5 \mathrm{~dB}$ (NR-relays) and $1.5 \mathrm{~dB}$ (R-relays) are obtained.

\subsection{Outage probability}

In this subsection, numerical results of the outage probability for the protocols are considered in statistically symmetric networks, e.g., $\Omega_{i j}^{2}=1$ and in small fixed $R$ regime, e.g., $R=1$. Figure 7 shows the outage probability of the DSTC and FDSTC protocols for NR-relays and R-relays as a function of $\mathrm{SNR}_{\text {norm }}$ which is defined by $\mathrm{SNR}_{\text {norm }}=\frac{P}{N_{0}} \frac{1}{\left[2^{R}-1\right]}[1]$. The analytic expressions perfectly match with the simulation results in high SNR region. The R-FDSTC protocol has better performance than the R-DSTC protocol when $\mathrm{SNR}_{\text {norm }}$ is greater than $21 \mathrm{~dB}$. Similarly, in the case of NR-relays, the FDSTC protocol provides better performance than the DSTC protocol when $\mathrm{SNR}_{\text {norm }}$ is greater than $30.5 \mathrm{~dB}$. This result is quite interesting since despite using more time slots for the transmission protocol (due to the data exchange between relays), the FDSTC protocol still reaches a better outage probability than the DSTC protocol in the high SNR region, no matter what type of relays is used as a result of higher diversity order of the FDSTC system as compared to the conventional DSTC.

The trade-off between the diversity order and the multiplexing gain of the systems is shown in Figure 8. The NRFDSTC system can achieve a maximum diversity order $(d(g)=3$ for a system with one source, two relays, and one destination). On the other hand, the diversity order of the NR-DSTC system is only two. For R-relays, while the DSTC system only gets the first diversity order, the FDSTC system can achieve the second diversity order. This means, in all cases (i.e., NR-relays or R-relays), using the FDSTC system can help to get higher diversity order. Moreover, there are some interesting sharp transitions in Figure 8 . We will use the FDSTC protocol for R-relays when $g<1 / 6$, otherwise when $g \geqslant 1 / 6$ the DSTC protocol is used; similarly for NR-relays, the FDSTC will be preferred for $g<1 / 8$.

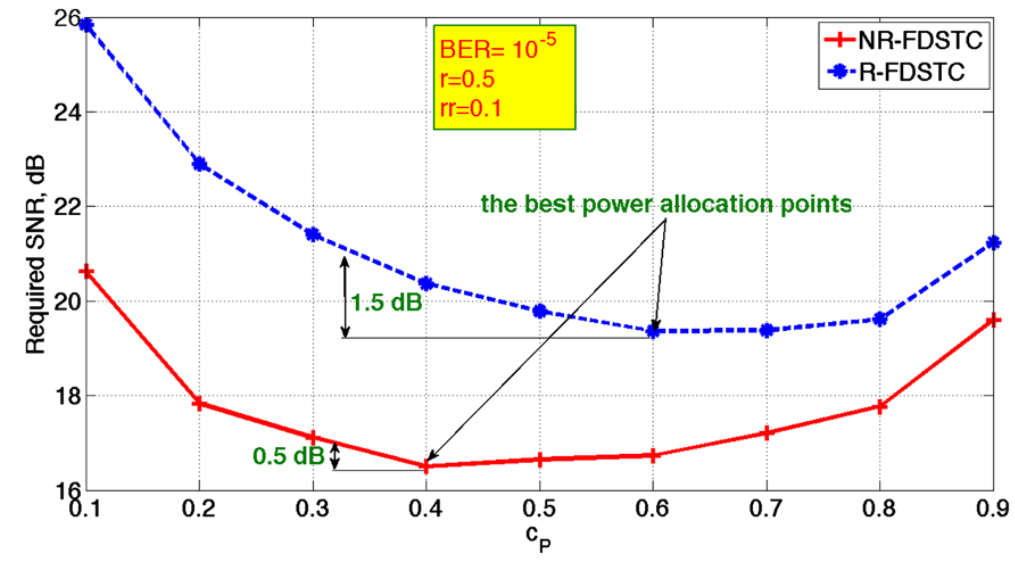

Figure 6 Optimal power allocation of the FDSTC protocols $(r r=0.1$ and $r=0.5)$. 




Figure 7 Outage probability versus $\mathrm{SNR}_{\text {norm }}$, small, fixed $R$ regime $(R=1)$ and for statistically symmetric networks, i.e., $\Omega_{i j}^{2}=1$.

\subsection{Energy efficiency}

Figure 9 shows the total energy consumption per bit of the DSTC and FDSTC protocols with NR-relays and Rrelays based on parameters of typical energy model [21] in two cases: (1) when the transmission distance $d_{\mathrm{sd}}<30$ $\mathrm{m}$ and (2) when $d_{\text {sd }}>100 \mathrm{~m}$. The case when $30 \mathrm{~m}<$ $d_{\text {sd }}<100 \mathrm{~m}$ is not illustrated since there is no cross-over point. At short-range transmissions, the FDSTC protocol consumes more energy than the DSTC protocol due to the data exchange between the relays. However, for longrange transmissions, the lower transmission energy of the FDSTC protocol makes it become energy-efficient. With R-relays, at $d_{\text {sd }}=25 \mathrm{~m}$, the FDSTC protocol can save up to $50 \%$ of the total energy consumption. Otherwise, with NR-relays, the FDSTC protocol consumes less energy than the DSTC only when $d_{\text {sd }}>200 \mathrm{~m}$ and at $d_{\text {sd }}=270$ $\mathrm{m}$, the FDSTC protocol can save $20 \%$ of the total energy consumption.

\section{Conclusion}

The main contribution of this paper is to propose and to evaluate the FDSTC protocol with NR-relays and Rrelays. A thorough analysis on the performance of the FDSTC protocol, followed by simulation results, is given to show its good performance. With two simple SISO transmissions added between the two relays, a significant SNR gain is obtained (i.e., $3.7 \mathrm{~dB}$ for NR-relays and $18.1 \mathrm{~dB}$ for R-relays). The relative distance of relays is shown to have a large impact on the required SNR. However, thanks to the FDSTC protocol, this impact can be significantly reduced by up to $75 \%$. In terms of outage probability, at the same spectral efficiency, the FDSTC protocol turns out to outperform conventional DSTC protocols in high SNR region. This is due to the fact that the FDSTC protocol helps to increase the diversity order of the system (full diversity in case of NR-relays). In terms of energy consumption, the FDSTC protocol is still more

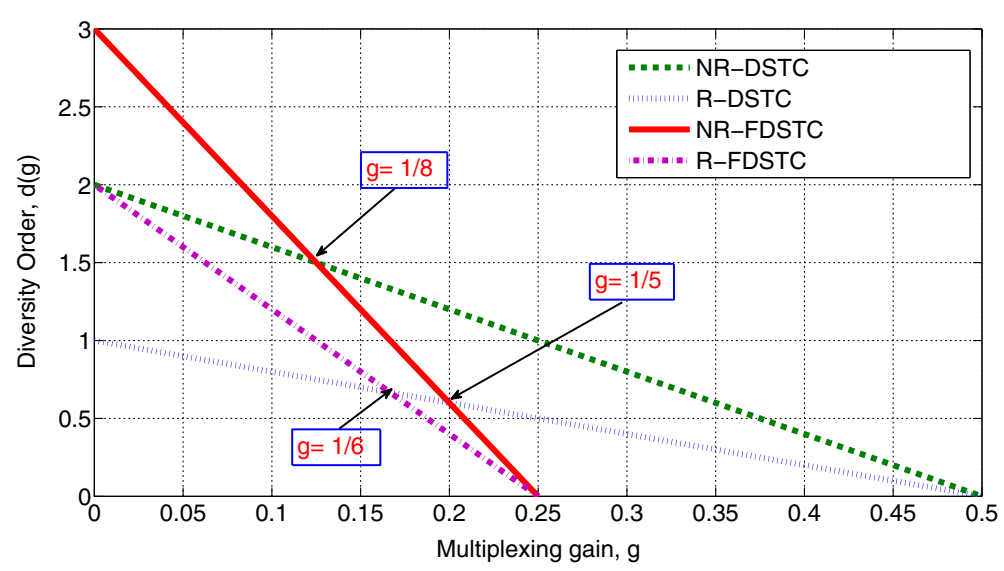

Figure 8 Diversity order $d(g)$ versus multiplexing gain $g$. 

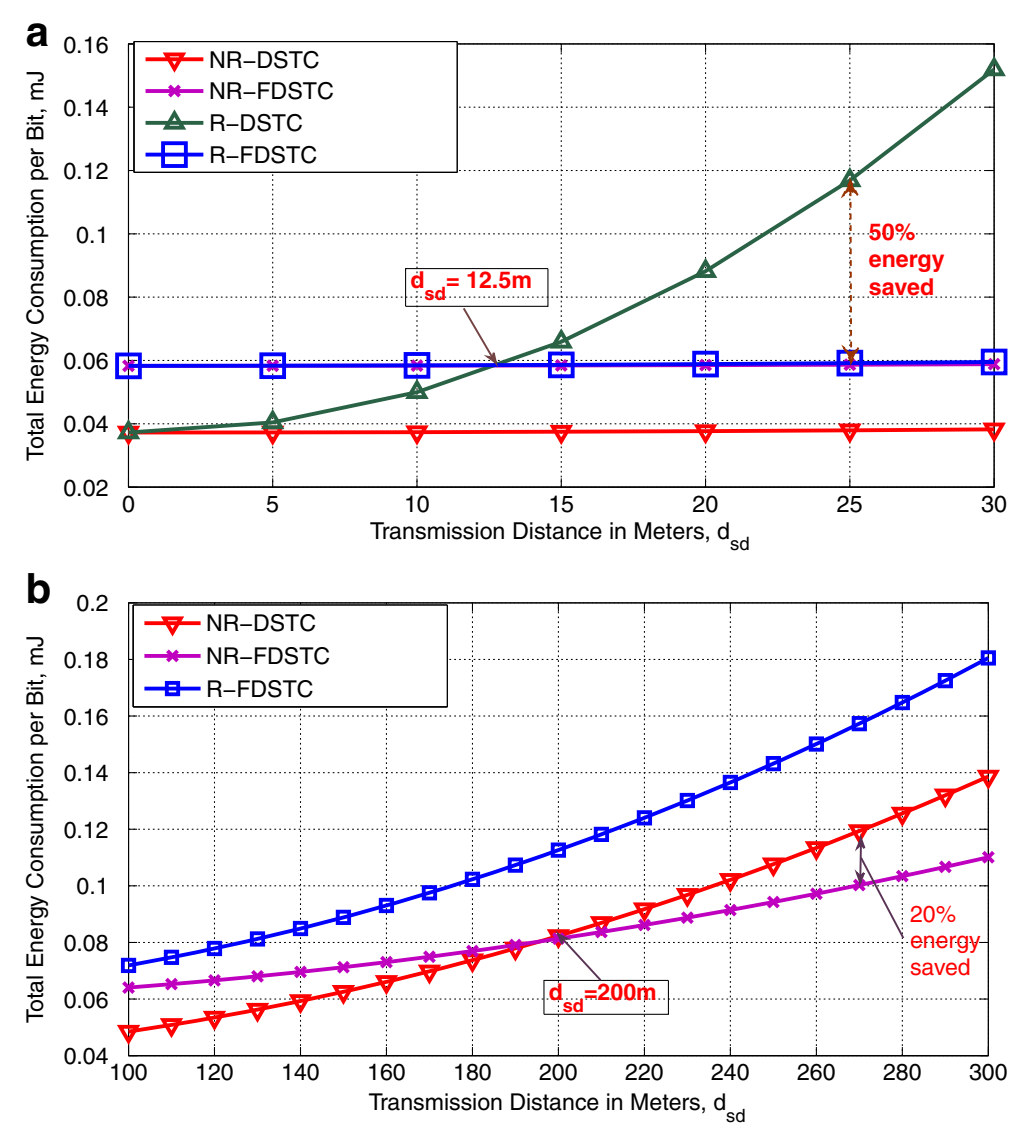

Figure 9 Total energy consumption per bit of the FDSTC and DSTC protocols with NR-relays and R-relays for two cases. (a) The transmission distance $d_{s d}<30 \mathrm{~m}$ and $(\mathbf{b})$ The transmission distance $d_{s d}>100 \mathrm{~m}$ ).

energy efficient than the DSTC protocol for long-range transmissions.

The good performance of the FDSTC protocol is clearly demonstrated in this paper using simple NR-relays and R-relays. Advanced techniques for relay networks (such as relay selection [15,16,31], optimum power allocation [32], etc.), which are used to improve the performance of DSTC systems, can be thoroughly considered for FDSTC systems as well. In the system where the number of relays is greater than two, the data exchange between the relays becomes very complicated. As a result, the spectral efficiency of the FDSTC protocol can be reduced due to the data exchange between relays. However, this problem can be solved by associating DSTC-based and repetition-based relaying protocols which is being studied.

\section{Appendix 1}

\section{Proof of (11)}

To approximate the closed-form ASEP of the NR-FDSTC protocol, we need to find the MGF of $\Gamma(v, t)=\frac{v t}{v+t+1}$, where $v$ and $t$ are two independent random variables with respective probability density functions (pdf) $f_{v}(v)=$ $\frac{1}{v_{0}^{2}} v e^{-\frac{v}{v_{0}}}$, and $f_{t}(t)=\frac{1}{t_{0}^{2}} t e^{-\frac{t}{t_{0}}}$.

The cumulative distribution function (cdf) of $\Gamma(v, t)$ is found for $\gamma>0$ based on [33]

$$
\begin{aligned}
F_{\Gamma}(\gamma)=1 & -2 \sqrt{\frac{\gamma^{2}+\gamma}{p}}\left(1+\frac{\gamma \sigma}{p}+\frac{2 \gamma^{2}+\gamma}{p}\right) \\
& \times e^{-\frac{\sigma}{p} \gamma} K_{1}\left(\frac{2}{\sqrt{p}} \sqrt{\gamma^{2}+\gamma}\right) \\
- & 2 \frac{\gamma^{2}+\gamma}{p}\left(1+\frac{\gamma \sigma}{p}\right) e^{-\frac{\sigma}{p} \gamma} K_{0}\left(\frac{2}{\sqrt{p}} \sqrt{\gamma^{2}+\gamma}\right),
\end{aligned}
$$

with $\sigma=t_{0}+v_{0}, p=t_{0} v_{0}$, and $K_{l}($.$) the modified Bessel$ function of the second kind with order $l$. 
The pdf of $\Gamma(v, t)$ is found by taking the derivative of $F_{\Gamma}(\gamma)$ and using (Equation 9.6.26 in [34])

$$
\begin{aligned}
f_{\Gamma}(\gamma)= & \frac{2}{p^{2} \sqrt{p}} \gamma \sqrt{\gamma^{2}+\gamma}\left(4 \sigma \gamma+\sigma^{2}+2 \sigma-2 p\right) \\
& \times e^{-\frac{\sigma}{p} \gamma} K_{1}\left(\frac{2}{\sqrt{p}} \sqrt{\gamma^{2}+\gamma}\right) \\
& +\frac{2}{p^{2}} \gamma\left(\left(\frac{\sigma^{2}}{p}+4\right) \gamma^{2}+\left(\frac{\sigma^{2}}{p}+4\right) \gamma+1\right) \\
& \times e^{-\frac{\sigma}{p} \gamma} K_{0}\left(\frac{2}{\sqrt{p}} \sqrt{\gamma^{2}+\gamma}\right) .
\end{aligned}
$$

The MGF is then derived by using its definition and using the change of variable $\gamma \rightarrow \gamma_{1}-1 / 2$

$$
\begin{aligned}
M_{\Gamma}(s)= & e^{\alpha / 2}\left[\frac { 2 } { p \sqrt { p } } \left(-\left(1-\frac{\sigma^{2}}{p}\right) \frac{\delta J_{0}}{\delta \beta}+\left(\frac{\sigma^{2}}{p}-\frac{2 \sigma}{p}-2\right)\right.\right. \\
& \left.\times \frac{\delta^{2} J_{0}}{\delta \alpha \delta \beta}-\frac{4 \sigma}{p} \frac{\delta^{3} J_{0}}{\delta \beta \delta \alpha^{2}}\right) \\
& +\frac{2}{p^{2}}\left(-\left(\frac{\sigma^{2}}{p}+4\right) \frac{\delta^{3} J_{0}}{\delta \alpha^{3}}-\frac{1}{2}\left(\frac{\sigma^{2}}{p}+4\right) \frac{\delta^{2} J_{0}}{\delta \alpha^{2}}\right. \\
& \left.\left.+\frac{\sigma^{2}}{p} \frac{\delta J_{0}}{\delta \alpha}-\frac{\sigma^{2}}{8 p} J_{0}\right)\right],
\end{aligned}
$$

where $\alpha=(\sigma-p s) / p, \beta=2 / \sqrt{p}$ and

$$
\begin{aligned}
J_{0}= & \int_{1 / 2}^{\infty} e^{-\alpha \gamma_{1}} K_{0}\left(\beta \sqrt{\gamma_{1}^{2}-(1 / 2)^{2}}\right)=\frac{1}{2 c}\left[e^{-\frac{c}{2}} E_{1}\left(\frac{\alpha-c}{2}\right)\right. \\
& \left.-e^{\frac{c}{2}} E_{1}\left(\frac{\alpha+c}{2}\right)\right]
\end{aligned}
$$

with $\left.c=\sqrt{\alpha^{2}-\beta^{2}}, \operatorname{Re}\{s\}<\sigma / p+2 / \sqrt{(} p\right)$, and $E_{1}($.$) the$ exponential integral function defined as (Equation 5.1.1 in [34]). Note that $J_{0}$ is derived by using the integrand in (Equation 646 in [35]).

The first and second order derivatives of $J_{0}$ with respect to $\alpha$ or/and $\beta, \frac{\delta J_{0}}{\delta \beta}, \frac{\delta^{2} J_{0}}{\delta \alpha \delta \beta}, \frac{\delta^{2} J_{0}}{\delta \alpha^{2}}$, and $\frac{\delta J_{0}}{\delta \alpha}$ can be found in [23]. The third order derivatives of $J_{0}, \frac{\delta^{3} J_{0}}{\delta \beta \delta \alpha^{2}}$, and $\frac{\delta^{3} J_{0}}{\delta \alpha^{3}}$ can be directly derived

$$
\begin{aligned}
\frac{\delta^{3} J_{0}}{\delta \alpha^{3}}= & \frac{\alpha}{c^{7}}\left(-3 \alpha^{2}-\frac{3}{2} \alpha^{2} c-\frac{9}{2} \beta^{2}-\frac{9}{4} \beta^{2} c-\frac{3}{8} \alpha^{4}+\frac{3}{8} \beta^{4}\right. \\
& \left.-\frac{1}{16} \alpha^{2} c^{3}\right) e^{-\frac{c}{2}} E_{1}\left(\frac{\alpha-c}{2}\right) \\
& +\frac{\alpha}{c^{7}}\left(3 \alpha^{2}-\frac{3}{2} \alpha^{2} c+\frac{9}{2} \beta^{2}-\frac{9}{4} \beta^{2} c+\frac{3}{8} \alpha^{4}\right. \\
& \left.-\frac{3}{8} \beta^{4}-\frac{1}{16} \alpha^{2} c^{3}\right) e^{\frac{c}{2}} E_{1}\left(\frac{\alpha+c}{2}\right) \\
& +\frac{1}{c^{6}}\left(\frac{5}{2} \alpha^{3}+11 \alpha^{2}+4 \beta^{2}+\frac{1}{2} \alpha^{4}-\frac{3}{2} \alpha^{2} \beta^{2}-\frac{5}{2} \alpha \beta^{2}\right. \\
& \left.+\frac{1}{4} \beta^{4}\right) e^{\frac{-\alpha}{2}}
\end{aligned}
$$

$$
\begin{aligned}
\frac{\delta^{3} J_{0}}{\delta \beta \delta \alpha^{2}}= & \frac{1}{c^{7}}\left(6 \beta \alpha^{2}+3 \alpha^{2} \beta c+\frac{5}{8} \alpha^{4} \beta-\frac{1}{2} \alpha^{2} \beta^{3}+\frac{3}{2} \beta^{3}\right. \\
& \left.-\frac{1}{8} \beta^{5}+\frac{3}{4} \beta^{3} c+\frac{1}{16} \alpha^{2} \beta c^{3}\right) e^{-\frac{c}{2}} E_{1}\left(\frac{\alpha-c}{2}\right) \\
& +\frac{1}{c^{7}}\left(-6 \beta \alpha^{2}+3 \alpha^{2} \beta c-\frac{5}{8} \alpha^{4} \beta+\frac{1}{2} \alpha^{2} \beta^{3}-\frac{3}{2} \beta^{3}\right. \\
& \left.+\frac{1}{8} \beta^{5}+\frac{3}{4} \beta^{3} c+\frac{1}{16} \alpha^{2} \beta c^{3}\right) e^{\frac{c}{2}} E_{1}\left(\frac{\alpha+c}{2}\right) \\
& +\frac{1}{c^{6}}\left(-13 \alpha \beta+\frac{1}{4} \alpha^{3} \beta-\frac{2 \alpha^{3}}{\beta}-\frac{\alpha^{4}}{\beta}-\frac{9}{2} \alpha^{2} \beta\right. \\
& \left.++\frac{3}{2} \beta^{3}-\frac{\alpha^{5}}{4 \beta}\right) e^{\frac{-\alpha}{2}}
\end{aligned}
$$

\section{Appendix 2}

\section{Proof of (16)}

In this appendix, the closed-form error probability of the $\mathrm{R}$-FDSTC protocol is derived based on the decoding of the combined signals at destination $y_{d}[2 k]$. Let $w_{2 k}=$ $\operatorname{Re}\left\{y_{d}[2 k]\right\}$, so

$$
\begin{aligned}
w_{2 k}= & \gamma_{s d} x[2 k]+\gamma_{r_{1}} d \hat{x}_{r_{1}}[2 k]+\gamma_{r_{2} d} \hat{x}_{r_{2}}[2 k] \\
& -\frac{\sqrt{\epsilon_{r_{1}} \epsilon_{r_{2}}} \operatorname{Re}\left\{h_{r_{1} d}^{*} h_{r_{2} d}\right\}}{N_{r_{2} d}} \hat{x}_{r_{1}}[2 k+1] \\
& +\frac{\sqrt{\epsilon_{r_{1}} \epsilon_{r_{2}}} \operatorname{Re}\left\{h_{r_{1} d}^{*} h_{r_{2} d}\right\}}{N_{r_{1} d}} \\
& \times \hat{x}_{r_{2}}[2 k+1]+z_{y_{d}}^{\prime}[2 k],
\end{aligned}
$$

where $z_{y_{d}}^{\prime}[2 k]=\operatorname{Re}\left\{z_{y_{d}}[2 k]\right\}$.

Let the following events be defined 


$$
\begin{aligned}
\mathcal{E}_{1,1} & \equiv\{x[2 k]=1, x[2 k+1]=1\} ; \quad \mathcal{E}_{1,-1} \equiv\{x[2 k] \\
& =1, x[2 k+1]=-1\} \\
\mathcal{E}_{-1,1} & \equiv\{x[2 k]=-1, x[2 k+1]=1\} ; \quad \mathcal{E}_{-1,-1} \equiv\{x[2 k] \\
& =-1, x[2 k+1]=-1\} \\
\mathcal{H}_{1} & \equiv\left\{\hat{x}_{r_{1}}[2 k]=x[2 k], \hat{x}_{r_{2}}[2 k]=x[2 k] ; \hat{x}_{r_{1}}[2 k+1]\right. \\
& \left.=\hat{x}_{r_{2}}[2 k+1]\right\} \\
\mathcal{H}_{2} & \equiv\left\{\hat{x}_{r_{1}}[2 k]=x[2 k], \hat{x}_{r_{2}}[2 k]=-x[2 k] ; \hat{x}_{r_{1}}[2 k+1]\right. \\
& \left.=\hat{x}_{r_{2}}[2 k+1]\right\} \\
\mathcal{H}_{3} & \equiv\left\{\hat{x}_{r_{1}}[1 k]=-x[2 k], \hat{x}_{r_{2}}[2 k]=x[2 k] ; \hat{x}_{r_{1}}[2 k+1]\right. \\
& \left.=\hat{x}_{r_{2}}[2 k+1]\right\} \\
\mathcal{H}_{4} & \equiv\left\{\hat{x}_{r_{1}}[2 k]=-x[2 k], \hat{x}_{r_{2}}[2 k]=-x[2 k] ; \hat{x}_{r_{1}}[2 k+1]\right. \\
& \left.=\hat{x}_{r_{2}}[2 k+1]\right\} \\
\mathcal{H}_{5} & \equiv\left\{\hat{x}_{r_{1}}[2 k]=x[2 k], \hat{x}_{r_{2}}[2 k]=x[2 k] ; \hat{x}_{r_{1}}[2 k+1]\right. \\
& \left.=-\hat{x}_{r_{2}}[2 k+1]\right\} \\
\mathcal{H}_{6} & \equiv\left\{\hat{x}_{r_{1}}[2 k]=x[2 k], \hat{x}_{r_{2}}[2 k]=-x[2 k] ; \hat{x}_{r_{1}}[2 k+1]\right. \\
& \left.=-\hat{x}_{r_{2}}[2 k+1]\right\} \\
\mathcal{H}_{7} & \equiv\left\{\hat{x}_{r_{1}}[2 k]=-x[2 k], \hat{x}_{r_{2}}[2 k]=x[2 k] ; \hat{x}_{r_{1}}[2 k+1]\right. \\
& \left.=-\hat{x}_{r_{2}}[2 k+1]\right\} \\
\mathcal{H}_{8} & \equiv\left\{\hat{x}_{r_{1}}[2 k]=-x[2 k], \hat{x}_{r_{2}}[2 k]=-x[2 k] ; \hat{x}_{r_{1}}[2 k+1]\right. \\
& \left.=-\hat{x}_{r_{2}}[2 k+1]\right\}
\end{aligned}
$$

The error probability, conditioned to the channel, is given by

$$
\begin{array}{r}
P_{2 k}=\frac{1}{4} \sum_{m=1}^{8} \mathcal{P}\left(\mathcal{H}_{m}\right) \times\left[\mathcal{P}\left\{w_{2 k}>0 / \mathcal{E}_{-1,1}, \mathcal{H}_{m}\right\}\right. \\
+\mathcal{P}\left\{w_{2 k}>0 / \mathcal{E}_{-1,-1}, \mathcal{H}_{m}\right\} \\
\mathcal{P}\left\{w_{2 k}<0 / \mathcal{E}_{1,1}, \mathcal{H}_{m}\right\} \\
\left.+\mathcal{P}\left\{w_{2 k}<0 / \mathcal{E}_{1,-1}, \mathcal{H}_{m}\right\}\right]
\end{array}
$$

where the probability of the event $\mathcal{H}_{m}$ depends on the error probabilities at relays $p_{2 k}^{j}$ and $p_{2 k+1}^{j}\left(j \in\left\{r_{1}, r_{2}\right\}\right)(17)$ and

$$
\begin{aligned}
\mathcal{P}\left\{w_{2 k}<0 / \mathcal{E}_{1,1}, \mathcal{H}_{1}\right\} & =\mathcal{P}\left\{\gamma_{r_{1} d}+\gamma_{r_{2} d}+\gamma_{\mathrm{sd}}+z_{y_{d}}^{\prime}(2 k)<0\right\} \\
& =\frac{1}{2} \operatorname{erfc}\left(\sqrt{\gamma_{r_{1} d}+\gamma_{r_{2}}+\gamma_{\mathrm{sd}}}\right) \\
& =\frac{1}{2} \operatorname{erfc}\left(\beta_{2 k}\right) .
\end{aligned}
$$

Using the same approach, we can derive the other terms in (41)

$$
\begin{aligned}
\mathcal{P}\left\{w_{2 k}<0 / \mathcal{E}_{1,-1}, \mathcal{H}_{1}\right\} & =\frac{1}{2} \operatorname{erfc}\left(\beta_{2 k}\right) ; \\
\mathcal{P}\left\{w_{2 k}<0 / \mathcal{E}_{1,1}, \mathcal{H}_{2}\right\} & =\frac{1}{2} \operatorname{erfc}\left(\delta_{2 k}\right) ; \quad \mathcal{P}\left\{w_{2 k}<0 / \mathcal{E}_{1,-1}, \mathcal{H}_{2}\right\} \\
& =\frac{1}{2} \operatorname{erfc}\left(\delta_{2 k}\right) ; \\
\mathcal{P}\left\{w_{2 k}<0 / \mathcal{E}_{1,1}, \mathcal{H}_{3}\right\} & =\frac{1}{2} \operatorname{erfc}\left(\lambda_{2 k}\right) ; \quad \mathcal{P}\left\{w_{2 k}<0 / \mathcal{E}_{1,-1}, \mathcal{H}_{3}\right\} \\
& =\frac{1}{2} \operatorname{erfc}\left(\lambda_{2 k}\right) ; \\
\mathcal{P}\left\{w_{2 k}<0 / \mathcal{E}_{1,1}, \mathcal{H}_{4}\right\} & =\frac{1}{2} \operatorname{erfc}\left(\phi_{2 k}\right) ; \quad \mathcal{P}\left\{w_{2 k}<0 / \mathcal{E}_{1,-1}, \mathcal{H}_{4}\right\} \\
& =\frac{1}{2} \operatorname{erfc}\left(\phi_{2 k}\right) ; \\
\mathcal{P}\left\{w_{2 k}<0 / \mathcal{E}_{1,1}, \mathcal{H}_{5}\right\} & =\frac{1}{2} \operatorname{erfc}\left(\beta_{2 k}+\theta_{2 k}\right) ; \quad \mathcal{P}\left\{w_{2 k}\right. \\
& \left.<0 / \mathcal{E}_{1,-1}, \mathcal{H}_{5}\right\}=\frac{1}{2} \operatorname{erfc}\left(\beta_{2 k}-\theta_{2 k}\right) ; \\
\mathcal{P}\left\{w_{2 k}<0 / \mathcal{E}_{1,1}, \mathcal{H}_{6}\right\} & =\frac{1}{2} \operatorname{erfc}\left(\delta_{2 k}+\theta_{2 k}\right) ; \quad \mathcal{P}\left\{w_{2 k}\right. \\
& \left.<0 / \mathcal{E}_{1,-1}, \mathcal{H}_{6}\right\}=\frac{1}{2} \operatorname{erfc}\left(\delta_{2 k}-\theta_{2 k}\right) ; \\
\mathcal{P}\left\{w_{2 k}<0 / \mathcal{E}_{1,1}, \mathcal{H}_{7}\right\} & =\frac{1}{2} \operatorname{erfc}\left(\lambda_{2 k}+\theta_{2 k}\right) ; \quad \mathcal{P}\left\{w_{2 k}\right. \\
& \left.<0 / \mathcal{E}_{1,-1}, \mathcal{H}_{7}\right\}=\frac{1}{2} \operatorname{erfc}\left(\lambda_{2 k}-\theta_{2 k}\right) ; \\
\mathcal{P}\left\{w_{2 k}<0 / \mathcal{E}_{1,1}, \mathcal{H}_{8}\right\} & =\frac{1}{2} \operatorname{erfc}\left(\phi_{2 k}+\theta_{2 k}\right) ; \quad \mathcal{P}\left\{w_{2 k}\right. \\
& \left.<0 / \mathcal{E}_{1,-1}, \mathcal{H}_{8}\right\}=\frac{1}{2} \operatorname{erfc}\left(\phi_{2 k}-\theta_{2 k}\right),
\end{aligned}
$$

where $\beta_{2 k}, \delta_{2 k}, \lambda_{2 k}, \phi_{2 k}$, and $\theta_{2 k}$ are given in (21). Note that we have $\mathcal{P}\left\{w_{2 k}<0 / \mathcal{E}_{1,-1}, \mathcal{H}_{m}\right\}=\mathcal{P}\left\{w_{2 k}>\right.$

$$
\mathcal{P}\left(\mathcal{H}_{m}\right)= \begin{cases}\left(1-p_{2 k}^{r_{1}}\right)\left(1-p_{2 k}^{r_{2}}\right)\left[\left(1-p_{2 k+1}^{r_{1}}\right)\left(1-p_{2 k+1}^{r_{2}}\right)+p_{2 k+1}^{r_{1}} p_{2 k+1}^{r_{2}}\right], & m=1 \\ \left(1-p_{2 k}^{r_{1}}\right) p_{2 k}^{r_{2}}\left[\left(1-p_{2 k+1}^{r_{1}}\right)\left(1-p_{2 k+1}^{r_{2}}\right)+p_{2 k+1}^{r_{1}} p_{2 k+1}^{r_{2}}\right], & m=2 \\ p_{2 k}^{r_{1}}\left(1-p_{2 k}^{r_{2}}\right)\left[\left(1-p_{2 k+1}^{r_{1}}\right)\left(1-p_{2 k+1}^{r_{2}}\right)+p_{2 k+1}^{r_{1}} p_{2 k+1}^{r_{2}}\right], & m=3 \\ p_{2 k}^{r_{1}} p_{2 k}^{r_{2}}\left[\left(1-p_{2 k+1}^{r_{1}}\right)\left(1-p_{2 k+1}^{r_{2}}\right)+p_{2 k+1}^{r_{1}} p_{2 k+1}^{r_{2}}\right], & m=4 \\ \left(1-p_{2 k}^{r_{1}}\right)\left(1-p_{2 k}^{r_{2}}\left[p_{2 k+1}^{r_{1}}\left(1-p_{2 k+1}^{r_{2}}\right)+\left(1-p_{2 k+1}^{r_{1}}\right) p_{2 k+1}^{r_{2}}\right],\right. & m=5 \\ \left(1-p_{2 k}^{r_{1}}\right) p_{2 k}^{r_{2}}\left[p_{2 k+1}^{r_{1}}\left(1-p_{2 k+1}^{r_{2}}\right)+\left(1-p_{2 k+1}^{r_{1}}\right) p_{2 k+1}^{r_{2}}\right], & m=6 \\ p_{2 k}^{r_{1}}\left(1-p_{2 k}^{r_{2}}\right)\left[p_{2 k+1}^{r_{1}}\left(1-p_{2 k+1}^{r_{2}}\right)+\left(1-p_{2 k+1}^{r_{1}}\right) p_{2 k+1}^{r_{2}}\right], & m=7 \\ p_{2 k}^{r_{1}} p_{2 k}^{r_{2}}\left[p_{2 k+1}^{r_{1}}\left(1-p_{2 k+1}^{r_{2}}\right)+\left(1-p_{2 k+1}^{r_{1}}\right) p_{2 k+1}^{r_{2}}\right], & m=8\end{cases}
$$


$\left.0 / \mathcal{E}_{-1,-1}, \mathcal{H}_{m}\right\}$, and $\mathcal{P}\left\{w_{2 k}<0 / \mathcal{E}_{1,1}, \mathcal{H}_{m}\right\}=\mathcal{P}\left\{w_{2 k}>\right.$ $\left.0 / \mathcal{E}_{-1,1}, \mathcal{H}_{m}\right\}$. That leads to all terms in (41) which therefore give rise to (16).

\section{Competing interests}

The authors declare that they have no competing interests.

\section{Received: 29 May 2013 Accepted: 16 September 2013} Published: 3 October 2013

\section{References}

1. JN Laneman, GW Wornell, DNC Tse, Cooperative diversity in wireless networks: efficient protocols and outage behavior. IEEE Trans Inf. Theory. 50(12), 3062-3080 (2004)

2. JN Laneman, GW Wornell, Distributed space-time-coded protocols for exploiting cooperative diversity in wireless networks. IEEE Trans. Inf. Theory. 49(10), 2415-2425 (2003)

3. S Barbarossa, G Scutari, Distributed space-time coding for multihop networks. IEEE Int. Conf. Commun. (ICC). 2, 916-920 (2004)

4. P Anghel, G Leus, M Kavehl, Multi-user space-time coding in cooperative networks. IEEE Int. Conf. Acoustics, Speech, Signal Proc. (ICASSP). 4, IV73-76 (2003)

5. M Wu, W Xue, D Wubben, A Dekorsy, S Paul, An improved inter-relay cooperation scheme for distributed relaying networks, International ITG Workshop on Smart Antennas (WSA), Dresden, 7-8 Mar 2012. (IEEE, Piscataway, 2012), pp. 62-69

6. U Tanoli, R Abbasi, Q Utmani, M Usman, I Khan, S Jan, Hybrid TDMA-FDMA based inter-relay communication in cooperative networks over Nakagami-m fading channel. International Conference on Emerging Technologies (ICET), Islamabad, 8-9 Oct 2012. (IEEE, Piscataway, 2012), pp. 1-5

7. Y Hu, KH Li, KC Teh, An efficient successive relaying protocol for multiple-relay cooperative networks. IEEE Trans. Wireless Commun. 11(5), 1892-1899 (2012)

8. F Librino, A Munari, M Zorzi, Inter-relay traffic through network coding in cooperative wireless ad hoc networks. IEEE Global Telecommunications Conference (GLOBECOM), Houston, 5-9 Dec 2011. (IEEE, Piscataway, 2011), pp. 1-6

9. LQ V Tran, O Berder, O Sentieys, Non-regenerative full distributed space-time codes in cooperative relaying networks, IEEE Wireless Commun. Netw. Conf. (WCNC), Mexico, 28-31 Mar 2011. (IEEE, Piscataway, 2011). 1529-1533

10. P Anghel, M Kaveh, Exact symbol error probability of a cooperative network in a Rayleigh-fading environment. IEEE Trans. Wireless Commun. 3(5), 1416-1421 (2004)

11. P Anghel, M Kaveh, On the performance of distributed space-time coding systems with one and two non-regenerative relays. IEEE Trans Wireless Commun. 5(3), 682-692 (2006)

12. B Maham, A Hjorungnes, G Abreu, Distributed GABBA space-time codes in amplify-and-forward relay networks. IEEE Trans. Wireless Commun. 8(4), 2036-2045 (2009)

13. Y Jing, B Hassibi, Distributed space-time coding in wireless relay networks. IEEE Trans. Wireless Commun. 5(12), 3524-3536 (2006)

14. G Scutari, S Barbarossa, Distributed space-time coding for regenerative relay networks. IEEE Trans. Wireless Commun. 4(5), 2387-2399 (2005)

15. J Hu, N Beaulieui, Performance analysis of decode-and-forward relaying with selection combining. IEEE Commun. Lett. 11(6), 489-491 (2007)

16. W Su, A Sadek, K Liu, SER performance analysis and optimum power allocation for decode-and-forward cooperation protocol in wireless networks. IEEE Wireless Communications and Networking Conference (WCNC), vol. 2. (IEEE, Piscataway, 2005), pp. 984-989

17. P A Anghel, G Leus, M Kaveh, Distributed space-time cooperative systems with regenerative relays. IEEE Trans. Wireless Commun. 5(11), 3130-3140 (2006)

18. M Vajapeyam, U Mitra, Performance analysis of distributed space-time coded protocols for wireless multi-hop communications. IEEE Trans. Wireless Commun. 9, 122-133 (2010)

19. D Gunduz, M Khojastepour, A Goldsmith, H Poor, Multi-hop MIMO relay networks: diversity-multiplexing trade-off analysis. IEEE Trans Wireless Commun. 9(5), 1738-1747 (2010)
20. H Wicaksana, S Ting, M Motani, Y Guan, On the diversity-multiplexing tradeoff of amplify-and-forward half-duplex relaying. IEEE Trans. Commun. 58(12), 3621-3630 (2010)

21. S Cui, A Goldsmith, A Bahai, Energy-efficiency of MIMO and cooperative MIMO techniques in sensor networks. IEEE J. Selected Areas in Commun. 22(6), 1089-1098 (2004)

22. R Fantini, D Sabella, M Caretti, Energy efficiency in LTE-advanced networks with relay nodes. IEEE 73rd, Vehicular Tech. Conf (VTC Spring), Yokohama, 15-18 May 2011. (IEEE, Piscataway, 2011), pp. 1-5

23. H Kim, T Chen, A study of energy efficient transparent relay using cooperative strategy. IEEE 73rd Vehicular Tech. Conf. (VTC Spring), Budapest, 15-18 May 2011. (IEEE, Piscataway, 2011), pp. 1-5

24. T Peng, R de Lamare, A Schmeink, Adaptive distributed space-time coding based on adjustable code matrices for cooperative MIMO relaying systems. Commun., IEEE Trans. PP(99), 1-12 (2013)

25. J Abouei, H Bagheri, A Khandani, An efficient adaptive distributed space-time coding scheme for cooperative relaying. Wireless Commun., IEEE Trans. 8(10), 4957-4962 (2009)

26. B Sirkeci-Mergen, A Scaglione, Randomized space-time coding for distributed cooperative communication. IEEE Trans. on Signal Process. 55(10), 5003-5017 (2007)

27. M K Simon, M S Alouini, Digital Communication over Fading Channels. (Wiley, New York, 2000)

28. L Zheng, D Tse, Diversity and multiplexing: a fundamental tradeoff in multiple-antenna channels. IEEE Trans. Inf. Theory. 49(5), 1073-1096 (2003)

29. HWan, JF Diouris, G Andrieux, Time synchronization for cooperative communication in wireless sensor networks. Wirel. Pers. Commun. 63(4), 977-993 (2012)

30. T D Nguyen, O Berder, O Sentieys, Impact of transmission synchronization error and cooperative reception techniques on the performance of cooperative MIMO systems. IEEE Int. Commun Conf. (ICC), China, 4601-4605 (2008)

31. $Y$ Jing, $H$ Jafarkhani, Single and multiple relay selection schemes and their achievable diversity orders. IEEE Trans. Wireless Commun. 8(3), 1414-1423 (2009)

32. Y Jing, H Jafarkhani, Relay power allocation in distributed space-time coded networks with channel statistical information. IEEE Trans. on Wireless Commun. 10(2), 443-449 (2011)

33. V Emanian, P A Anghel, M Kaveh, Multi-user spatial diversity in a shadow-fading environment. IEEE Vehicular Tech. Conf. (VTC). 1, 573-576 (2002)

34. M Abramowitz, I A Stegun, Handbook of Mathematical Functions with Formulas, Graphs, and Mathematical Tables, 9th edn. (Dover Publications, New York, 1990)

35. I S Rappaport, I M Ryzhik, Table of Integrals, Series and Products, 5th edn (Academic Press, San Diego, 1994)

\section{doi:10.1186/1687-1499-2013-239}

Cite this article as: Tran et al.: On the performance of distributed space-time coded cooperative relay networks based on inter-relay communications. EURASIP Journal on Wireless Communications and Networking 2013 2013:239.

\section{Submit your manuscript to a SpringerOpen ${ }^{\circ}$ journal and benefit from:}

- Convenient online submission

Rigorous peer review

- Immediate publication on acceptance

- Open access: articles freely available online

- High visibility within the field

- Retaining the copyright to your article

Submit your next manuscript at $>$ springeropen.com 